\title{
5. Der Lichtgott der alten Letten
}

5.1. Seit dem vorigen Jahrhundert gab es, angefangen mit Auniņš, immer wieder Versuche, das Wesen von Ūsiņš linguistisch zu bestimmen. Wie wir gesehen haben, sind diese bis jetzt erfolglos geblieben. Hier muß dennoch ein ernsthafter Versuch erwähnt werden, in die Zusammenhänge, die sich hinter dem Namen von Ūsiņš verbergen könnten, Einblick zu gewinnen. Einen solchen Versuch hat Schmid unternommen. Er gibt zu, daß die bisherigen Ansätze erfolglos waren: ,Deshalb soll versucht werden, einen Weg zu gehen, der in lautlicher, grammatischer und semasiologischer Hinsicht einwandfrei zu sein scheint." 1

5.1.1. Schmid geht von der Annahme aus, daß der lettische $\bar{U} \operatorname{sings~und~}$ der russische усень urverwandt sind, wobei er ausführt: ,Lett. $\bar{u}$ und russ. $y$ lassen sich dann nur unter der Vorform lett. $u n$-, slav. $Q$ - vereinigen. Das $s$ ist in diesem Falle in beiden Sprachen zweideutig: es kann idg. - $\varepsilon$ - ebenso wie $-\hat{k}$ - repräsentieren. Damit ergeben sich die zwei Paare I *uns-/*ons/und/ *unk-/*onki-" "2 Nach Überprüfung des ersten Paares verwirft er dieses und wendet sich der zweiten Möglichkeit zu, indem er ausführt: ,Auch im Slavischen kann $Q$ sowohl auf *on als auch auf $*_{n}$ zurückgehen, worauf abulg. doti, dzme ,blasen" (russ. dutb) und auch grnati : apreuß. guntwei ,treiben" weisen können (Būga). Unter dieser Voraussetzung läßt sich

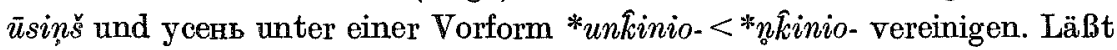
man des Suffix zunächst beiseite, dann ergibt sich zur Schwundstufe $* n \hat{k}$ eine Normalstufe *en $\hat{k}$. Übersetzt man dies wiederum ins Germanische, ergibt sich *ing-. Und damit wird man unmittelbar auf den im Germanischen nur noch in Spuren nachweisbaren Gott Ing geführt, über den W. Krause das Wesentliche gesagt hat ${ }^{[1]}$. Der Name selbst erscheint im angelsächsischen Runenlied als Ing, ihm entspricht der gotische Runenname für $\chi$ engruz in der Salzburg-Wiener Alcuin-Handschrift. Von ihm abgeleitet ist der Name der Inguaeones in der plinianischen Überlieferung und die zahlreichen Personennamen wie Inguiomerus, Ingibjorg, Ingimundr, Ingivaldr, ahd. Ingulf, Inguhilt etc. Im Nordischen sind auch Formen mit Grammatischem Wechsel belegt: Ingvarr/Ívarr, die W. Krause auf *Ingwia-harjaz/ * Inhwia-harjaz zurückführt ${ }^{[2]}$. Inhaltlich gesehen sind die Übereinstim-

3 Schmid 135.

2 Ib. 
mungen so, wie sie nicht besser sein könnten. Ing wird später von Frey, einem ausgesprochenen Fruchtbarkeitsgott, verdrängt. Sein Beiname ist Ingvi-Freyr! Wenn die kreisförmige Ing-Rune zunächst wirklich ein Symbol der Sonne war, ergibt sich eine weitere Beziehung zu $\bar{U} \operatorname{sinş~(in~Lied~Nr.~[38]~}$ - siehe oben - sind Sonne und Mond Söhne des $\bar{U}$.); ebenso durch die Anbringung des Runenzeichens an einem Hochzeitshaus bzw. in einer Dreschtenne. Als Grundlage der Personennamen nimmt W. Krause den

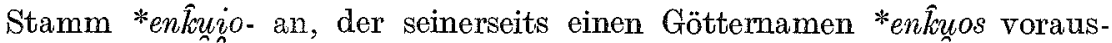
setzt, und diesen verbindet er überzeugend mit toch. A ónk, Beinkwe,,Mann" ${ }^{\text {"[3] }}$.

Diese Etymologie paßt zu dem labais vīrs $\bar{U}_{\text {sings }}$ ausgezeichnet. Somit bleibt nur noch die Frage offen, ob sich auch die Stammbildung des Götternamens im Baltischen und Slavischen in Einklang bringen läßt. Auf baltischer Seite bestehen keine Schwierigkeiten. Das Verhältnis von *enk̂no zu *nk̂kinio- läßt sich hinsichtlich des Ablauts z. B. mit lit. er̃dvas ,geräumig" - lett. irdens ,locker", lit. kraũjas „Blut" - kruvinis „blutig" vergleichen. Daß im Baltischen vor dem Suffix lett. -inžs, lit. -inis von $u$ oder wo-Stämmen keine Spur mehr zu finden ist, wird auch an anderen Beispielen deutlich. So gehört zu lett. tirgus „Markt" ein tirdziňš, zu lit. saldìs „,süß" saldinis, zu statùs ein statinis. Die Verschiedenheit der Bedeutung im Lettischen und Slavischen läßt auf unabhängigen Suffixantritt schließen. Das im Lettischen beliebte Suffix ist auch hier - sei es mit der Bedeutung ,hieber Üsinšs", sei es ohne Funktion - an den Stamm $\bar{u} s$ - angetreten $^{[4]}$. Im Slavischen aber läßt sich das Suffix - snjo- in seiner Grundbedeutung fassen. *ycémь (день) wäre der zum Gotte *en $\hat{k}(u)$ - gehörige (Tag). Offen bleiben muß die Frage, ob man ein altes Nebeneinander von

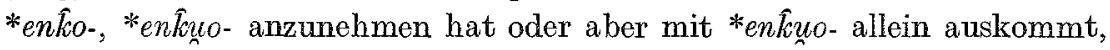
das erst im Baltischen (vgl. lit. gausà, gausvà „Überfluß", Adj. lit. gausùs, aber lett. gauss) und im Slavischen, wo die u-Flexion im Adjektiv ganz geschwunden ist, im Substantiv langsam zurückgedrängt wurde, ein *nkkuozu *nko- wurde. Die Wahrscheinlichkeit und die Methode sprechen für die letztere Alternative.

Wir möchten also glauben, daß man für das Germanische, Baltische and

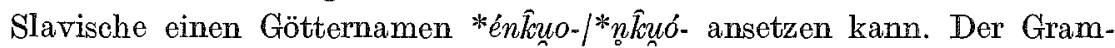
matische Wechsel im Germanischen und der Ablaut verraten ein noch älteres, nicht notwendigerweise athematisches Paradigma ${ }^{[5]}$. Der Name wird durch toch. eikkwe "Mann" gedeutet und der Guttural durch das Baltische und Slavische als $-\hat{k}$ - bestimmt. Damit wird ein Labiovelar ausgeschlossen, so daß nur eine Verbindung - $\hat{k} u$ - in Frage kommt, die zugleich die Normalentwicklung der Nasalis sonans zu -in- im Baltischen bzw. - - im Slavischen gestört haben mag. Diesen Namen trug ein Fruchtbarkeitsgott, der Frühling, Leben und Gedeihen brachte. Sein Kult läßt sich 
im Germanischen nur noch in Spuren, im Slavischen gar nicht und im Baltischen nur mehr hinter der christlichen St. Georgs-Verehrung nachweisen." "1

Das wichtigste an dieser Analyse von Schmid ist, daß er eine glaubhafte Begründung zur Lösung des bisher zwar erkannten, aber erfolglos untersuchten Problems des etymologischen Zusammenhangs des lettischen Usiņš mit dem russischen ycens geboten und eine weitere Stützung im System der indoeuropäischen Sprachen gefunden hat. Damit wird jetzt einerseits die ablehnende Haltung von Endzelīns gegenüber einer etymologischen Verbindung von Ūsiņ̌s mit aues-, andererseits die ständige Berufung auf den Zusammenhang zwischen Ūsiņ lich. Die Analyse von Schmid zeigt, daß dieser Zusammenhang von anderer Art ist, als man bisher behauptete. Es liegt außerhalb meiner Möglichkeiten, diese Ansicht kritisch zu bewerten. Interessant ist Hinweis Schmids auf die Ansicht von Krause über den germanischen Gott Ing und damit auf den Zusammenhang von Ūsiņš mit einem Fruchtbarkeitskult. Aber darüber werden wir uns erst nach einer weiteren Betrachtung unseres Materials unter diesem Aspekt äußern können.

5.1.2. Das zweite linguistische Problem, das eng mit dem Verständnis vom Wesen des Ūsinšs verbunden ist, aber bisher nicht erörtert wurde, bildet der in der Nachricht von Stribiņš vom Jahre 1606 erwähnte Ausdruck

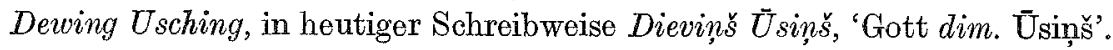
Man kann darüber verschiedener Meinung sein, wie diese Wortgruppe aufzufassen ist. Sie soll wohl zum Verständnis rom Wesen des Ūsiņ dienen. Diese Wörter sind in einen in lateinischer Sprache geschriebenen Bericht eingefügt. Da ein weiterer lettischer Kontext fehlt, mangelt eine sichere Grundlage zum richtigen Verständnis. Dazu kommt noch die in jener Zeit unbestimmte und unsichere Setzung der Satzzeichen, in diesem Fall des Kommas. Mit dem Ausdruck Dieviņš Usin̋š verbinden sich zwei Fragen: Haben die Letten, über die Stribiņš schreibt, tatsächlich selbst diesen Ausdruck gebraucht und hat Stribinšs ihn nur niedergeschrieben, oder handelt es sich um eine Konstruktion von Stribinšs selbst zur Charakterisierung des $\bar{U}$ siņ̧̌s? Ist gar diese Wortgruppe so zu verstehen, daß $\bar{U}_{\text {sinz }}$ die Apposition zu Dieviņšs bildet und zwischen beiden Worten nur ein Komma fehlt?

${ }^{1} \mathrm{Ib}$. Schmid hat zu diesem Zitat die folgenden Anmerkungen gemacht: ,[1.] Krause 1944, 229 bis 254. Das Folgende nach W. Krause. [2.] Vielleicht könnte man den a. a. O. 241 erwähnten Ungvinus rex Gothensium und das runeninschriftl. uywinaR (Ende 6. Jh.) auf die schwundstufige Form * $n k$ - zurückführen um den $w$-Umlaut nicht allzu früh datieren zu müssen? [3.] Krause 1944, 251. Vgl. Krause 1960, 57, 93, und auch B. yakwe, A yuk 'Pferd' ekuos. [4.] Beachte den einmal bezeugten Genitiv üsa! [5]. VgI. das Nebeneinander von got. waúrd, apreuß. wirds (<*urdho-) neben lit. var̃ das (*uordho-), lat. verbum ("uerdho-). 
Grammatikalisch gesehen, hat man es bei Dieviňs $\check{U}$ sin̆š mit einer Wortgruppe zu tun, bei der das Substantiv im Mittelpunkt steht. Wenn zwei Substantive nebeneinander stehen und sich im gleichen Kasus befinden, in diesem Fall im Nominativ, kann es sich um ein Attribut oder eine Apposition handeln ${ }^{1}$. In unserem Fall würde das klar sein, wenn zwischen beiden Wörtern ein Komma stünde. Sonst ergeben sich bei der Klärung des Verhältnisses beider Wörter zueinander Schwierigkeiten. Über solche Wortgruppen sagt Aina Bergmane: „Da beide Komponenten Hauptwörter sind und im gleichen Kasus stehen, kann man nur nach der sprachlich semantischen Natur beider Hauptwörter feststellen, welches von ihnen die unabhängige und welches die abhängige Komponente ist. ${ }^{62}$ Wenn man sich von dieser Erkenntnis leiten läßt, ist unschwer zu bestimmen, daß der Eigenname $\bar{U} \operatorname{sings~die~unabhängige~Komponente~in~dieser~Wortgruppe,~das~}$ Gattungswort Dieviņš die abhängige Komponente darstellt. Ferner hat sie zu solchen Sprachbildungen eine Anmerkung gemacht: „, Das begriffliche Verhältnis zwischen beiden Hauptwörtern ist das einer Apposition oder eines Beisatzes: solche Gruppen bezeichnen nicht nur eine Erscheinung und deren Merkmale, sondern drücken auch eine Synthese beider Vorstellungen oder Begriffe aus, mit anderen Worten - zwischen den Komponenten besteht sowohl eine attributive als auch eine prädikative Beziehung. ${ }^{63}$ Daß das in vollem Maße auf unsere Wortgruppe zutrifft, ist klar zu erkennen. Beide Wörter unserer Wortgruppe sind eine Synthese eingegangen zur Bezeichnung eines neuen Begriffs Dieviňš $\bar{U}$ sinš. Daraus ist ferner zu folgern, daß diese Konstruktion dem lettischen Sprachgebrauch entspricht, d. h. daß sie nicht unter dem Einfluß einer fremden Sprache entstanden ist. In diesem Zusammenhang gewinnt die Anwendung eines Satzzeichens sekundäre Bedeutung, denn die Wortgruppe selbst beinhaltet eine Apposition oder richtiger, schließt eine solche nicht aus. Dem begrifflichen Inhalt nach ist $\bar{U}$ siņš als unabhängiges Hauptwort das $N$. proprium und Dieviņš das $N$. appellativum. Praktisch würde das bedeuten, daß Stribiṇ̌s damit sagen will, die Letten hätten einen Gott angebetet, der sich Ūsiņš nannte ${ }^{4}$. Und wie wir im lateinischen Text Iasen, war das Deus equorum.

1 Cf. Endzelinns 1951, 1023, § 820.

2 Bergmane, Aina 68.

3 Ib. $68 \mathrm{~s}$.

4 Unter unseren Texten findet sich auch eine V., die zu 11 beigefügt ist:

$\bar{U}$ siņš dieviņš slaist $\bar{\jmath} j \bar{a} s$;

Mana stallla pakal̦ā.

$E j, \bar{U} \sin$, stalliti $\overline{\text {, }}$

Baro labus kumelinus.
Ūsiñ Gott dim. richtete sich hoch auf, hinter meinem Pferdestall. Geh, Usin, in den Pferdestall dim., füttere die Rößlein, daß sie gut werden.

In diesem Text finden wir die umgekehrte Wortfolge „Ūsinšr Gott" dim., Es gibt nur 
Doch mit dieser Erkenntnis über den Inhalt der Bezeichnung ist die Frage nicht beantwortet, ob Stribiņš sie so aufgeschrieben hat, wie er sie im Volk gehört hatte, oder ob es sich um seine eigene Sprachschöpfung handelt. Wenn man sich vom Kontext leiten läßt, in dem diese Wortgruppe erscheint, und von dem Umstand, daß der ganze Text lateinisch geschrieben ist, in den diese zwei Wörter lettisch eingefügt sind, möchte man meinen, daß Stribiṇš gerade betonen und unterstreichen wollte, daß er diese Wörter so gehört habe. Anders ausgedrückt — es handelt sich um Originalwörter, d. h. um ein Zitat. Doch ergeben sich gewisse Schwierigkeiten, wenn man den ganzen Text liest, in dem Ūsinš zusammen mit anderen Göttern auftritt: Deo equorum, quem vocant Dewing Uschinge offerunt 2 solidos et duas panes et frustum pinguedinis, quam in ignem conjiciunt. Deo Moschel, qui est vaccarum deus, offerunt butyrum, lac, caseum, et, si quae infirmatur vacca, statim ad arbores illisque offerunt et liberatur. Deo agrorum frumentorumque, quem vocant Dewing Cerehlicing, certis temporibus bovem nigrum, gallinam nigram, porcellum nigrum, etc. et aliquot tonnas cerevisiae, prout illos deus Cerehling iuverit, plus vel minus offerunt in silvis ${ }^{1}$. Wenn man diesen Text näher betrachtet, stellt sich heraus, daß von verschiedenen Göttern die Rede ist. Unser Ausdruck erscheint im Text in besonderer syntaktischer Form: Deo equorum, quem vocant Dewing Uschinge. Im laufenden Text gibt Stribin̄š in lateinischer Sprache bekannt, daß die Letten einem besonderen Pferdegott opfern, den er mit dem speziellen lettischen Ausdruck näher erklären will. Hier hätte ein Wort genügt - der Eigenname Üsiņ̌s. Aber er fügt noch das Wort Dewing hinzu. Wie wir gesehen haben, erscheint im selben Text eine ganz ähnliche, parallele Konstruktion: Deo agrorum frumentorumque, quem vocant Dewing Cerehlicing. Auch hier hätte zur Erklärung ausgereicht, daß der Gott der Felder auf lettisch Cerehlicing geheißen hat. Aber Stribinš hat auch hier Dewing hinzugefügt. Nur als vom dritten Gott die Rede ist, ändert sich die Konstruktion: Deo Moschel, qui est vaccarum deus ${ }^{2}$. Die beiden ersten hier erwähnten Götter sind mit ihren Eigennamen zusammen mit dem erklärenden Gattungswort Dewing genannt. Im dritten Fall wird nur der lettische Name der Göttin der Kühe angegeben. Das führt zu der Frage, weshalb dieser Unterschied gemacht wird. Ist es nur Zufall? Vielleicht nur eine Stilvariation? Es scheint, daß es sich nicht nur um einen Zufall handelt. Wenn die Wissenschaftler mit einen solchen Text den der bekannte Textverfasser Tortuss eingesandt hat, deshalb braucht man ihm vielleicht keine größere Aufmerksamkeit zuzuwenden. Dievs bildet in diesem Fall die Apposition zu Ūsinšs, doch das ändert inhaltlich nichts am vorhin Gesagten.

1 Fontes 2, 106.

2 Die Bedeutung des Wortes Moschel ist unklar; ich habe mich dazu in anderem Zusammenhang ausführlicher geäußert. Cf. Biezais 1955, 76, 265 ss. 
ihren Behauptungen recht haben, daß es sich bei Moschel um ein verderbtes lettisches Wort handelt, dem Marša oder die hl. Maria, in der Volksetymologie Mära, entsprichtı, dann hatte Stribiņš gar keine Möglichkeit, in der Volkssprache den Ausdruck Dewing (Dieviňs) in Verbindung mit Moschel zu hören. Moschel ist ein Wesen weiblichen Geschlechts, während es sich bei Dieviņš um ein Deminutiv männlichen Geschlechts handelt. Dieses konnte man mit einem Wesen weiblichen Geschlechts nicht verbinden. Daß Stribiņš nicht recht begriffen hat, wer mit Mosehel gemeint war, zeigt der Umstand, daß er von Moschel als einem Wesen männlichen Geschlechts spricht - deus, Deo Moschel anstelle von Deae Moschel. Es ist klar, daß seiner unrichtigen Auffassung nicht die Volkssprache zugrunde lag und auch nicht zugrunde liegen konnte, denn diese kannte keinen „,Dieviņš Moschel". Diese Erkenntnis bestätigt ferner, daß dort, wo Stribiṇs in seinem lateinischen Text den Ausdruck Dewing gebraucht, er diesen auch tatsächlich gehört hat.

Bei der Datierung dieser Konstruktionen in das Jahr 1606 ergeben sich andere Schwierigkeiten. Dievs und dievinz als Gattungsnamen haben eine späte semantische Bedeutung, die sich unter dem Einfluß der christlichen Mission gebildet hat. Der ursprüngliche Sinn des Wortes dievs ist Himmel. Dieses Wort wird auch in anderer semantischer Bedeutung gebraucht, nämlich zur Bezeichnung Gottes in der vorchristlichen Religion der Balten, und zwar als Eigenname ( $N$. proprium $)^{2}$. Erst unter dem Einfluß des Christentums gewinnt dieser einen neuen Sinn als Gattungsname zur allgemeinen Bezeichnung Gottes. So wurde der Ausdruck offenbar von Stribiņ. bzw. von denen benutzt, wo er ihn gehört hatte.

Mit dieser Feststellung stoßen wir auf neue Schwierigkeiten. Wenn das Wort $\bar{U}$ siņš den Eigennamen eines vorchristlichen Gottes darstellt, dann ergibt sich eine ganz eigenartige Situation. Diesem vorchristlichen Gott ist als Erklärung die unter dem Einfluß des Christentums entstandene semantische Bedeutung des Wortes dievs 'Gott' beigefügt. Im Synkretismus ist das möglich und verständlich. Aber es macht uns doch sehr vorsichtig in der Beurteilung des Namens $\bar{U} \operatorname{sinss,~insbesondere,~wenn~man~an~die~}$ verschiedenartigen und widersprüchlichen Erklärungen denkt. Hier ist noch an Beobachtungen in bezug auf andere lettische vorchristliche Götter zu erinnern. Die mit Eigennamen genannten Götter: Saule, Laima, Mēness u. a., tragen in der Volkstradition gewöhnlich nicht auch den Gattungsnamen dievs. Aber wo er vorkommt, ist es ein untrügliches Zeichen, daß das später unter dem Einfluß des Christentums geschehen ist. Dasselbe

\footnotetext{
1 Cf. ib.; Adamoviěs 1937, 71.

2 Der Erforschung dieser Frage im lettischen religiösen Synkretismus habe ich eine besondere Monographie gewidmet. Cf. besonders 1961, 13 ss.
} 
gilt für die Wortgruppe dieviņš $\bar{U}$ singš. Stribiņš hat in seinem Text Dewing mit einem großen Buchstaben geschrieben, als ob es ein Eigenname sei. Zweifellos hat er das unter dem Einfluß der deutschen Rechtschreibung für Substantive getan.

Dieser Sachverhalt hilft auch, die Entstehungszeit der hier dargestellten Sprachschöpfung zu bestimmen. Sie konnte nur nach Beginn der christlichen Mission Ende des 12. und im 13. Jh. entstanden sein. Wenn man weiß, daß der christliche Glaube durch die Eroberer im Laufe eines Jahrhunderts formal aufgezwungen wurde, ohne ernsthaftes Bemühen, ihn im Volke zu verwurzeln, muß man folgern, daß die Umwandlung des alten Gottes Dievs zu einem Gattungswort noch später stattgefunden haben wird, aber jedenfalls nicht später, als Stribiņš es in seinem Bericht an das Jesuitenkollegium in Riga gebraucht hat. Die linguistischen Untersuchungen zeigen, daß man bei der Bestimmung vom Wesen des Usiņ̌ die erste Komponente der Wortgruppe dievinš $\bar{U}$ siņš beiseite lassen kann, da sie zur Erklärung des ursprünglichen Wesens dieses Gottes nichts beiträgt.

5.2. Die linguistischen Forschungen zur Lösung des Problems des Usiṇš haben ihre Bedeutung, aber sie genügen für sich allein nicht und können keine Lösung bieten. Im Laufe dieser Arbeit haben wir verschiedene Standpunkte kennengelernt und haben sie im Lichte des zugänglichen Quellenmaterials kritisch betrachtet. In diesem Abschnitt haben wir auch festgestellt, daß Schmid es war, der die Diskussion über das Problem des Ūsin̨š a uf dem Gebiet der Linguistik vorangetrieben hat.

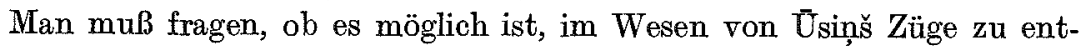
decken, die bisher noch unbemerkt geblieben sind. Um auf diese Frage zu antworten, muß man sich noch einmal dem Teil des Materials zuwenden, a uf das wir vorhin schon hingewiesen haben.

5.2.1. Dankbares Material stellt unsere oben erwähnte Gruppe von 26 Liedern dar. Wir haben davon - dank der Forschungen von Kreics 6 Texte ausgewählt, in denen von einem bewaffneten Mann die Rede war. Kreics lehnte auch Texte mit Dieviņš ab, da er sie für Plagiate hielt. Wir haben bereits nachgewiesen, daß das Problem viel komplizierter ist. Daher wollen wir uns zuerst den beiden Texten zuwenden, in denen Dieviņš erwähnt wird $(30063,1 ; 34067,6)$. In Wirklichkeit muß man von einem einzigen Text sprechen, denn beide Lieder unterscheiden sich nur durch ein Wort in der 2. Zeile, das unterschiedlich geschrieben wird, nämlich akmenoju $(33063,1)$ und akmenaju $(34067,6)$ :

$P a$ zālūti Dievinš brauca

Akmenoju kumelinu;

Tas atveda kokiem lapas,

Zemei zalu ābolinu. $\quad$ 34067, 6
Über das Gras dim. fuhr Dieviṇš mit einem steinernen RoB dim.; er brachte den Bäumen Blätter, der Erde grünen Klee. 
Wie wir bereits wissen, ist in dieser ganzen Gruppe in der zweiten Hälfte der Lieder davon die Rede, daß sich Blätter und grünes Gras einfinden wie immer die Namen der Spender auch lauten mögen: Ūsiņš, Dieviņš, ein bewaffneter Mann u. a. Daher kann nur die Analyse der ersten zwei Zeilen der Lieder hier und weiterhin einige Klarheit bringen. In diesem Text ist die Rede von Dieviṇš. Schon das allein, daß dieser Text für sich dasteht, wenn auch in 2 Varianten, kann zu dem Entschluß führen, ihn von der Untersuchung auszuschließen. Er könnte Zufallscharakter haben. Doch gleich ist dazu zu bemerken, daß das vielleicht voreilig gehandelt sein könnte, insbesondere, wenn man diesen Text mit anderen vergleicht, in denen Dieviņš in denselben Funktionen wie Ūsinšs erscheint. Es genügt hier, den folgenden Text kennenzulernen.

Te gulējši pieguḷnieki,

Te kūruši uguntinu,

Te Dievinš sildäjies

Te palicis mêtelītis. $\quad 30074$
Hier haben die Nachthüter geschlafen, hier haben sie ein Feuerchen geschürt, hier hat Dievs dim. sich gewärmt, hier ist das Mäntelchen geblieben.

Das ist ein bekanntes Nachthüterlied, zudem recht verbreitet (10 V.). Es sagt aus, daß am Feuer der Nachthüter zusammen mit diesen auch Dievin̄š sich gewärmt habe. Es ist wahr, gerade einen solchen Text, in dem Ưsin̦š anstelle von Dieviņš erwähnt wäre, gibt es nicht. Daher könnte man meinen, daß auch dieser Text nur Zufallscharakter habe. Aber das ist nicht so. Groß ist die Anzahl der Texte, in denen Ūsiņš und Dieviņš nebeneinander in verschiedenen Varianten anzutreffen sind. Wir lesen im Lied 47, wo in dunkler Nacht die Pferde zur Nachthütung hinausgelassen werden, die Bemerkung: „Nun, Usin, steht in deiner Gewalt, nun in deiner Hand." Wörtlich wird dasselbe im Text 30201 gesagt, nur steht hier anstelle von Ūsinšs Dieviņš. Wir hörten, daß man von Ūsiņš Pferde kaufen kann (54, 57, 61), man kann sie aber auch von Diers kaufen (10476, 29628, 2, 4-5), ebenso kann man sie von Dievs leihen (29608, 29653). Mit Ūsiņs kann man Pferde tauschen (18), aber dasselbe gilt auch für Dievs (29608). Wir lasen mehrfach in den Texten, daß Ūsiņš zur Nachthütung reitet (48), doch nicht weniger ausdrucksvoll sind die Texte, die von Dieviņš auf dem Weg zur Nachthütung berichten.

Kliedza vīri, zviedza zirgi,

Dieviñš jāja pieguḷă;

Dziedāj' meitas, māva govis, Māra laida ābulā. 738
Die Männer schrien, die Pferde wieherten, Dievs dim. ritt zur Nachthütung; die Mädchen sangen, die Kühe muhten, die hl. Maria ließ im Klee weiden.

Ähnlich 29176, 54785 u. a.

In der Variante des Liedes 29176 werden die Nachthüter Dieva piegul,- 
nieki, Nachthüter des Dievs, genannt, womit ein übriges Mal die enge Verbundenheit von Dievs mit ihnen aufgezeigt wird. So wird das Bild von Dievs, der sich am Feuer der Nachthüter wärmt, besser verständlich.

Zum Abschluß dieser Übersicht über das Auftreten von Dieviṇš in den Liedertexten über Ũsinš ist auf den Text 30055 hinzuweisen:

Dievs iemauktus šlcindināja

Man $\ddot{a}$ stalla diben $\bar{a}$,

Sukādams, glaudìdams

Manus bērus kumelinus. 30055
Dievs klimperte mit dem Zaumzeug hinten in meinem Stalle, er striegelte, er glättete meine braunen Rößlein.

Inhaltlich stimmt dieser Text mit dem vorhin erörterten Lied 46 überein ${ }^{\mathbf{1}}$. Ein Einblick in diese Texte und deren Vergleich zeigen, daß Dieviņš neben Ūsinšs in den gleichen Funktionen eines Pferdepatrons auftritt. Daher ist es begründet, die Behauptung von Kreics abzulehnen, daß es sich bei der Einführung von Dieviņš in diese Lieder um ein Plagiat handele. Das konnte ihm so scheinen, da er sich nur mit einem einzigen Text beschäftigt hat.

Die Frage erhält ein anderes Schwergewicht, wenn Dieviňš in diesen Texten so oft auftritt. Das zwingt zur Frage: wie ist es zu erklären, daß Ūiņ̄̌ und Dieviņš in vielen Funktionen nebeneinander stehen? Sind sie identisch? Falls sie nicht identisch sind, wie erklärt sich diese Erscheinung? Als ich seinerzeit das Problem des altlettischen Dievs erörterte, blieb ich bei der Auffassung, daß es sich bei Dievs in diesen Texten um den altlettischen Himmelsgott Dievs handelte ${ }^{2}$.

Die weiteren Forschungen zeigten, daß auch Pērkons, der Donnergott, unstreitig mit dieviņš bezeichnet wird:

Dieviňš rüc, Dieviňš rüc,

Zibšnus met ozolā;

Labāk met ozolā,

Ne bālina sētmalie. $\quad 33700$
Gott dim. grollt, Gott dim. grollt, schleudert Blitze in die Eiche; wirf [sie] lieber in die Eiche, als an den Hofrand des Brüderleins.

Es gibt eine größere Anzahl solcher Texte (27766, 33724, 33704, 4 u. a.). Das bedeutet, daß in den Volkstraditionen Pêrkons und Dieviņš als identisch bezeichnet werden müssen ${ }^{3}$. Das wirft auch ein Licht auf meine Erkenntnisse, die ich bereits in meinen Forschungen über den altlettischen Dievs geäußert habe, der, wie wir eben gesehen haben, in der Rolle des Ūsiňš, insbesondere zur Zeit der Nachthütung, auftritt. So haben wir es in Wirklichkeit mit der Erscheinung zu tun, daß nebeneinander unter demselben Namen drei verschiedene Wesen bekannt sind: der altlettische Himmelsgott Dievs, in der Verkleinerungsform Dieviņš, der Donnergott

1 Die eben erwähnten Texte mit Dieviṇš sind in anderem Zusammenhang eingehender behandelt worden. Cf. Biezais 1961, 146 ss.

2 Ib. 148.

3 Cf. Biezais 1972,163 . 
Përkons, der auch mit Dieviņš bezeichnet wird, und Ūsiņ̌s, der bereits im Text von Stribiņš Dieviņš genannt wird. Im letzten Fall stellten wir fest, daß diese Bezeichnung unter dem Einfluß des Christentums entstanden ist. Dasselbe ist auch über die Benennung von Pērkons als Dieviň̌ zu sagen¹. Wenn dem so ist, muß man im Zusammenhang mit den beiden letzteren vermuten, daß in den Texten, in denen wir oben Dieviņš anstelle von Ūsiņš fanden, vielleicht ursprünglich nur Üsiņš gestanden hat. So wie Dievinğ in die Texte von Përkons Eingang fand und den ursprünglichen Pērkons verdrängte, konnte das auch mit den Texten über Ūsiņš geschehen sein. Auf Grund meiner Forschungen aus letzter Zeit bin ich bereit, die Möglichkeit zuzulassen, daß in der Gruppe der Usiņš-Texte in zweien, in denen Dieviņš vorkommt, ursprünglich Ūsiṇš gestanden haben könnte. Doch kann ich mich mit völliger Sicherheit darüber nicht äußern². Beider Funktionen jedoch sind die gleichen.

5.2.2. Noch weitere Zusammenhänge werden beim Vergleich von 5 Texten mit dem Namen Ūsiņš mit den drei übrigen Gruppen sichtbar, in denen Dieviņš (2mal), ein bewaffneter Mann (6mal) und namentlich nicht genannte Personen (13mal) auftreten. In allen Liedern über Üsiņš wird in der ersten Zeile gesagt, daß Ūsiņš ein Reiter ist, zum anderen, daß er über den Berg reitet, und drittens ebenso konsequent in der 2. Zeile, daß er ,mit einem Rößlein aus Stein" reitet. Was zeigen die übrigen drei Gruppen? In zwei Texten fährt Dieviņš jeweils ,über das Gras". Doch sein Pferd ist auch hier aus Stein. Der bewaffnete Mann reitet in allen 6 Texten ebenso wie Úsins. Doch ist in keinem der Texte die Rede von einem Reiten über einen Berg. In 4 Texten wird nur die Tatsache erwähnt, daß er herbeireitet, und in zweien, daß er über einen großen Acker reitet. In 4 Texten wird erwähnt, daß er ein steinernes Rößlein reitet. Bevor wir den Vergleich der ŪsiñšLieder mit der letzten Gruppe - der der anonymen Reiter - fortsetzen, wollen wir das bisherige Ergebnis betrachten: Usiňš und der bewaffnete Mann sind in allen Texten Reiter. Daß der bewaffnete Mann bzw. der hl. Georg Reiter sind, ist verständlich, daher bieten die Texte in dieser Hinsicht keinen Unterschied zu den Usinš-Texten. Dievs ist stets ein Fahrer. Ebenso konsequent ist Úsiņš niemals ein Fahrer. Ein aufmerksamer Leser wird auf den Text 45 hinweisen, aber diesem werden wir uns später zuwenden. Der bewaffnete Mann ist ebenso wie Üsiňs niemals ein Fahrer. Auch der hl. Georg ist kein Fahrer. Schließlich stellt sich heraus, daß Ūsiņš

\footnotetext{
1 Ib. $109 \mathrm{~s}$.

2 Das bedeutet insbesondere, daß auch die in meiner Arbeit vom Jahre 1961 behandelten ähnlichen Texte, in denen Dieviņš erwähnt wird, sich auf Ūsiņš beziehen könnten. Doch bringen sie inhaltlich nichts Neues zur Erforschung des Problems des Üsiņ̌ in dieser Arbeit.
} 
und Dieviņš stets auf einem steinernen Pferd reiten, während wir diese Aussage über den bewaffneten Mann in 4 von 6 Texten finden.

So gewinnen wir das Bild, daß Ũsinšs allein als Reiter über einen Berg, Dieviņš allein als Fahrer auftritt. Ūsinšs und Dieviņš reiten auf einem steinernen Pferd, aber nur zum Teil ist das bei dem bewaffneten Mann der Fall. Dieviņš fährt stets über den Acker, auch der bewaffnete Mann erscheint auf dem Acker.

Welches Bild ergibt sich, wenn man die Ũsiņš-Lieder mit der Gruppe von 13 Liedern vergleicht, in denen anonyme Wesen auftreten? Zunächst werden diese mit verschiedenen unbestimmten Ausdrücken bezeichnet. Wenn im Text eine rhetorische Frage steht, wird das unbestimmte Wesen in 8 Fällen mit dem Fürwort , der" (30063, 3; 34067; -, 4-5, LTdz 1, 2556), in 3 Fällen $(30063,1 ; 34067,2 ;-, 8)$ mit dem unbestimmten Fürwort ,einer" bezeichnet. In je einem Fall ist die Rede von einem steinernen Mann $(34067,1)$ oder einem alten Mann $(30063,2)$. Es ist bezeichnend, daß die Texte in überwiegender Mehrheit (9) vom Herreiten dieser Wesen reden, es handelt sich also um Reiter (34067; -, 1-2; -, 4; -, 8; LTdz 1, 2556). Nur gelegentlich ist die Rede auch von einer anderen Art des Herbeikommens, nämlich in einem Fall vom Herlaufen $(30063,1)$ und in zwei Fällen vom Kommen $(30063,3 ; 34067,5)$. Über den exsten dieser Texte haben wir uns schon früher geäußert; er muß als unbedeutend beiseite gelassen werden. So verringert sich die Zahl der zu betrachtenden Texte auf 12 .

Bedeutsam sind gerade die Texte, die von jemandes Kommen sprechen. Aus der 2. Zeile geht hervor, daß der Betreffende einen grauen Mantel trägt $(30063,3 ; 34067,5)$. Aber an diesem grauen Mantel erkennen wir auch seinen Eigentümer - es ist Dieviņšs. Wir haben schon früher den Unterschied zwischen Dievin̦š und anderen Laubbringern festgestellt. Er ist der einzige, der gefahren kommt, zudem über grünes Gras. In dieser Liedergruppe wird jetzt seine Tätigkeit nur durch ,er kam"s ausgedrückt. Das bietet Grund zu der Behauptung, daß zwischen diesen Laubbringern eine klare Trennung vorzunehmen ist - auf der einen Seite die Reiter Usinšs und der bewaffnete Mann (der hl. Georg), auf der anderen Seite Dieviņš, der Fahrer und Kommende. Hier ist zur Vermeidung von Mißverständnissen zu bemerken, daß auf lettisch nālct 'kommen' von verschiedener Bedeutung sein kann, nämlich zu Fuß kommen oder ganz allgemein sich einfinden bedeutet, wobei über die Art und Weise nichts ausgesagt wird ${ }^{2}$. Unser Text läßt vermuten, daß das letztere gemeint ist. Aber das ändert nichts an den aufgezeigten Unterschieden.

1 Über diese Attribute von Dievs habe ich mich an anderer Stelle geäußert. Cf. Biezais 1961, 100.

${ }^{2}$ Cf. ME 2,698 ss. 
Bei Fortsetzung der Betrachtung dieser 12 Texte stellt sich ferner heraus, da $\beta$ in keinem von ihnen weder das Reiten noch das Kommen lokalisiert sind, wie das in der vorhin erwähnten Gruppe der Fall war, wo als Ort des Geschehens ein Berg und ein Acker genannt wurden. Dagegen wird eine reichhaltige Information darüber gegeben, mit welchen Fortbewegungsmitteln diese Wesen sich einfinden. In 10 von unseren 12 Texten wird zu Pferde geritten. Eine Ausnahme bilden nur die bereits bekannten 2 Texte, in denen jemand in einem grauen Mantel kommt. Dieser letzte Umstand vertieft noch die erwähnte Kluft zwischen Reitenden und Fahrenden bzw. Kommenden und bekräftigt das oben Gesagte.

$\mathrm{Zu}$ noch mehr Klarheit führt das Kennenlernen der Pferde der Reiter. Auf die erwähnten 10 Texte verteilen sich folgende Pferde: 4 steinerne (30063, 1; 34067, 2; - 4), eines aus Silber (34067, 1), eines aus Stahl (34067); in den übrigen Texten wird statt dessen die Farbe der Pferde erwähnt drei sind rauchfarben (34067, LTdz 1, 2556) und eines ist grau $(34067,8)$. Während wir vorläufig die Frage des steinernen. Pferdes zurückstellen, lohnt es sich, noch einige andere dieser Texte einzusehen. Es bedarf keines großen Scharfsinns, um zu verstehen, daß ein Pferd aus Stahl in den Rahmen der Volkstradition nicht hineinpaßt. Im Lettischen ist das Wort terauds 'Stahl' aus dem. Kivischen entlehnt'. Das bringt auf den Gedanken, daB es sich mit Vorstellungen von einem bewaffneten Mann verbinden läßt. Hier wäre auch gleich über das silberne Pferd zu sprechen; wir werden gleich darauf zurückkommen. Zunächst ist ein anderer Umstand zu beachten. Das ist die in den Texten offen hervortretende Tendenz, an der Vorstellung vom. Steinernen auch da festzuhalten, wo das nicht nötig wäre, wo es vielmehr widersprüchlich ist. Reitet der unbekannte Reiter ein graues Pferd, dann trägt dieses ,einen steinernen Sattel auf dem Rücken“ $(34067,8)$. Weshalb? Selbst wenn der zu dieser Textgruppe gehörige Dieviňs über das Gras fährt, dann tut er es seltsamerweise mit einem ,,steinernen Rößlein“ $(34067,5)$, das ebenso wie das Pferd mit dem steinernen Sattel auf dem Rücken aus dem Rahmen der Wirklichkeit fällt. Ja, selbst der bewaffnete Mann, unser hl. Georg, reitet auf einem "steinernen RöBlein" herbei $(34067,7)$. Welcher Zusammenhang besteht zwischen dem mit einer eisernen Rüstung gepanzerten hl. Georg und dem unmöglichen steinernen Pferd? Um dies Bild vollkommener zu machen, wenden wir uns dem silbernen Pferd zu:

Akmentina vīrinš jāja

Sudrabina zirdzin $\bar{x}$, Kokiem lapas skaitĩdams, Auguminus mērīdams. 34067, 1

${ }^{1}$ Cf. ME 4, 172; auch zeps 189 ss.
Ein steinemes Männerchen ritt auf einem silbernen Pferdchen, er zählte die Blätter der Bäume, maß den Wuchs dim. 
Das steinerne Pferd hat sich in ein silbernes Pferd verwandelt, und der unbekannte Reiter ist zum steinernen Reiter geworden, einer neuen Erscheinung neben Ūsin̄š, Dieviņš und dem christlichen Heiligen. Es ist nicht schwer, die Bedeutung dieses Textes für unser darzustellendes Problem zu erkennen. Es ist eine Bestätigung, zudem in schöner, freier dichterischen Phantasie. Damit könnte man vielleicht alle unsere Texte von steinernen Pferden als Ausdruck schöpferischer Phantasie ansehen und unsere ganze vorangegangene Analyse als gegenstandslos betrachten. Ich würde gern diesem voreiligen Kritiker zustimmen, das ist aber nicht möglich, und zwar aus mehreren Gründen.

Hier muß die grundsätzliche Frage der poetischen Ausdrucksweise religiöser Erlebnisse und überhaupt des Zusammenhangs zwischen Religion und Poesie beiseite gelassen werden, da ich mich darüber bereits wiederholt an anderer Stelle geäußert habe ${ }^{1}$. In erster Linie muß man sich hier der ständigen Erscheinung des steinernen Pferdes, die auftritt, wo sie nicht hingehört, zuwenden. Man darf die alten Letten nicht für naiver halten als sie waren und deshalb nicht denken, daß sie in ihrem Leben und ihren Vorstellungen das Mögliche und Unmögliche nicht unterscheiden konnten, zumindest in so einfachen Zusammenhängen, wie sie mit einem steinernen Pferd verbunden sind. Die poetische Ausdrucksweise und die dabei verwendete symbolische Sprache will einen Inhalt ausdrücken, den es sonst nicht gibt. So sind die Vorstellungen vom steinernen Pferd anzusehen und zu bewerten. Sie finden ihre Berechtigung und Begründung in der Ganzheit des Weltbildes. Das Wort Weltbild führt uns zum Mittelpunkt dieses ganzen Vorstellungskomplexes, nämlich zum steinernen Himmel. Weiter gelangen wir im Verlauf der Gedanken zu folgendem.

Der steinerne Himmel ist eine sehr alte und verbreitete indoeuropäische Vorstellung. Wie er sich in den lettischen Traditionen spiegelt, habe ich in einem besonderen Forschungsbericht dargestellt².

1 Cf. Biezais $1955,65 \mathrm{~s} ; 1972,213 \mathrm{~s}, 474 \mathrm{~s}$; auch James 102.

2 Cf. Biezais 1960, 11 ss, and 1972, 236 ss. Danach sind mehrere Arbeiten erschien en die diese Ansicht weiter bekräftigen: ef. Maher 450, $457 \mathrm{~s}$; Lazzeroni, $118 \mathrm{~s}$, ist zum folgenden Ergebnis gekommen: „E' giunto il momento di trarre le conclusioni: esse sono tre e presentato un grado decrescente di certezza: A) è certo che il significato di 'cielo' non è mai appartenuto al sser. áśman- né, tanto meno, al suo ascendente indoeuropeo: esso consegue ad una innovazione 'centrale' caratteristica di un'area, grecoiranica anche altrimenti individuata, coerente col rinnovamento dei nomi del cielo avvenuto in un gruppo di lingue di cui il greco e l'iranico fanno parti. Questa conclusione ha conseguenze anche per l'etimologia: una volta, infatti, negata l'origine indoeuropea dell'equivalenza 'pietra' = 'cielo' e stabilita l'area e le fasi dell'innovazione, la connessione, sostenuta da alcuni, del nome germanico del cielo (got. himins ecc.) col nome i.e. della pietra appare anche per questo motivo, difficilmente ammissibile. B) E' probabile che l'innovazione abbia avuto origene dalla defigurazione di una metafora 
Die Vorstellung vom steinernen Himmel ist daher auch die Grundlage, auf der die Frage nach dem steinernen Pferd zu lösen ist. Aber in diesem Zusammenhang ist noch ein anderer Umstand zu berücksichtigen. Es stimmt, wie unsere Analyse zeigte, daß alle in unserer Liedergruppe erwähnten Wesen Reiter sind, mit Ausnahme von Dievs. Das heißt nicht, daß Dievs in den lettischen Traditionen nicht als Reiter bekannt wäre. Ganz im Gegenteil, er ist der Eigentümer von Pferden und auch Reiter ${ }^{1}$. Wenn er in unseren zwei Texten nicht als Reiter erwähnt wird, so ändert das nichts an der Tatsache. Jetzt verstehen wir besser, warum es den christlichen Reitern, dem hl. Georg u. a., leicht gelang, in die Usinšš-Lieder Eingang zu finden. Dieses Moment ist besonders im Zusammenhang mit den anonymen Laubbringern zu beachten.

Weitere Klarheit zur Lösung des Problems bringt ein Blick auf die Umwelt, in der das Reiten stattfindet. In allen 5 Texten reitet Ūsiņš stets über einen Berg. Ebenso konsequent hat keiner der anonymen Reiter es mit einem Berg zu tun. Es ist verständlich, daß auch der bewaffnete Mann, d. h. der hl. Georg, nicht über einen Berg reiten kann². Es ist auch bezeichnend, daß Dieviņš in unseren Texten über das Gras fährt. Hier ist ferner auch auf die Präposition hinzuweisen, mit der das Reiten lokalisiert wird. Es wird durch die Präposition pār 'über' (36, LTdz 1, 2557) ausgedrückt. In zwei Texten $(30063,34067,6)$ steht par. Letztere Schreibweise ist durch die ungenaue Bezeichnung langer Vokale im lettischen Schrifttum des vorigen Jahrhunderts zu erklären. Hier ist jedoch daran zu erinnern, daß Auninšs, ungeachtet der beiden zu allererst veröffentlichten Texte, besonders angemerkt hat, daß das Wort mit einem langen Vokal zu schreiben sei, und, entsprechend der Orthographie jener Zeit, pahr geschrieben hat. Welches ist der Inhalt der Vorstellung: $\bar{U}$ siņš jāja pār kalniņu, 'Úsiņš ritt über den Hügel'? Damit lassen sich verschiedene Vorstellungen verbinden. Einerseits, daß er über den Hügel hinüberreitet, andererseits, da $\beta$ er auf dem Hügel selbst reitet. Und schließlich kann es bedeuten, daß er, über caratteristica di un mito preario in cui la pietra era assunta a simbolo della nube; quando il nome della nube passó a significare il cielo anche l'antico nome della pietra, ormai divenuto sinonimo di nube, avrà subito la stessa sorte. C) E' possibile che un'eco di quiesta vicenda risuoni anche in greco. Ma qui l'indagine deve arrestarsi: la scarsità dei dati darebbe spazio più all'immaginazione che alla storia. " Es ist zu bedauern, dass Lazzeroni das lettische Material nicht kennt (ef. Biezais 1960, $8 \mathrm{ss}$ ).

I Über Dievs als Reiter ef. Biezais 1961, 96 ss.

2 Das hier erwähnte Reiten über den Berg ist nicht zu verwechseln mit dem Reiten im Himmel. Auch die christlichen Heiligen sind als Reiter auf dem Pferde im Himmel bekannt. Gelegentlich ist auch Jesus zu einem solchen Reiter geworden, nicht auf dem Esel, wie man es erwarten könnte, sondern auf dem Pferde (cf. Strzygowski 55, Abb., und 58). Verständlicherweise ist das unter dem Einfluß alter persiseher Traditionen geschehen. 
den Berg reitend, sich nähert, sichtbar wird, da ist. Der Inhalt unserer Texte läßt das letztere annehmen, denn gleichzeitig mit seinem Reiten über den Berg findet sich Gras ein. In alltäglicher Sprache ausgedrückt — sein Reiten über den Berg ist ein Synonym für seine Ankunft. Wenn wir uns ferner zweier Dinge erinnern, daß mit dem Berg in diesen Texten der Himmelsberg gemeint ist, daß die eindrucksvollste Reiterin und Fahrerin auf diesem Berg Saule ist ${ }^{1}$ und neben ihr die anderen Himmelsgötter: Pērkons, Mēness, Dieva dēli², dann haben wir einen sicheren Grund für die Behauptung gewonnen, daß Ūsiņ̌s ursprünglich zu dieser Familie der Himmelsgötter gehört hat.

Auf Grund dieser Feststellung können wir jetzt überprüfen, wie das mit Ūsinšs, der auf einem steinernen Pferd reitet, in Einklang zu bringen ist. Ich habe schon darauf hingewiesen, daß man nicht glauben müsse, der alte Lette sei so naiv gewesen, einen Reiter, selbst einen Gott, mit einem steinernen Pferd in Verbindung zu bringen. Das steinerne Pferd ist eine Metapher, zudem eine solche, die sich richtig ins Gesamtbild eines steinernen Himmels einfügt. Man kann viele Vermutungen darüber anstellen, welche Assoziationen oder psychischen Vorgänge im weiteren Sinne hinter der Herausbildung solcher Vorstellungen stehen mögen. Aber das ist eine Frage, die von Forschern zu lösen ist, die sich mit den kreativen Funktionen der menschlichen Seele, und nicht nur auf dem Gebiet der Religion, beschäftigen. Es handelt sich um eine sprach- und religionspsychologische Frage. Das von Ūsiṇš gerittene steinerne Pferd steht zwar in groteskem Widerspruch zur. Wirklichkeit des Lebens, aber nicht zur religiösen Gesamtschau der Welt. Im Gegenteil, dieser Widerspruch verhilft dazu, den eigentlichen Platz von Ūsinšs unter den Himmelsgöttern zu bestimmen.

Wie tief Usinšs in der Familie der Himmelsgötter verwurzelt und mit ihr verwachsen ist, zeigen unsere oben behandelten 26 Texte. Nach der gewonnenen Erkenntnis über Ūsiņš, den Reiter auf steinernem Pferd, können wir uns diesen Texten wieder zuwenden und Antwort auf die Fragen geben, die so lange offen gelassen werden mußten. Wir bemerkten in ihnen ein ständiges Festhalten an der Vorstellung vom steinernen Pferd. So bietet sich das unerklärliche Bild, daß Dieviņš über das Gras fährt und dabei ein steinernes Pferd benutzt $(34067,6)$. Wenn man noch versuchen könnte, das damit zu erklären, daß Dieviņ̌s zur Familie der Himmelsgötter gehört, so ist dies ganz unmöglich in bezug auf den hl. Georg. Wie tief und einflußreich das Eindringen dieses christlichen Reiters an die Stelle von Usiņš auch gewesen sein mag, wobei er viele seiner Eigenschaften annahm, in

1 Näheres über die Bewegung von Saule über den Himmelsberg steht bei Biezais 1972, 238; cf. auch 1961, 90 ss.

2 Cf. ib. 71 ss, $111 \mathrm{ss}, 423$ ss. 
einer Hinsicht mußte er sich Ūsiņ š unterwerfen, er mußte das steinerne Pferd des Ūsiňs übernehmen. Das groteske Bild des in eine Rüstung gekleideten Mannes, der auf einem steinernen Roß reitet, wird dennoch unter den gegebenen Umständen verständlich. Der Umbildungsprozeß des Synkretismus ging weiter. Wir kennen heute nicht die eigentlichen Gründe; vielleicht geschah es im vorigen Jahrhundert, als unsere Texte aufgeschrieben wurden. Das wirft noch einmal ein neues Licht auf das so schwer zu lösende Problem vom bewaffneten Mann. Es war schwer, dem in der Mentalität des Christentums befangenen Letten mit offenen Worten zu sagen, daß der hl. Georg ein steinernes Pferd reitet. Aber nichts stand entgegen, den hl. Georg durch den bewaffneten Mann zu ersetzen. Diese Vorstellung war dann doch leichter mit der Metapher vom steinernen Pferd in Einklang zu bringen. Das war weniger Anstoß erregend als ein hl. Georg auf einem steinernen Pferd. Das war die eine Art der Lösung. So wurde die vertraute vorchristliche Metapher vom steinernen Himmel bzw. vom steinernen Pferd bewahrt.

Aber es gab noch einen anderen Weg. Und auch diesen zeigen unsere Texte auf. Wenn man Ūsiņš durch den hl. Georg, den hl. Martin, den hl. Michael und im weiteren Verlauf auch durch einen bewaffneten Mann ersetzen konnte, was hinderte dann, noch einen Schritt weiter zu gehen? Und so kommen wir zu unserer Gruppe von 12 Liedern mit anonymen Texten, wo in rhetorischer Frage an Stelle von Ūsiņš ein fragendes Fürwort - ,wer" - getreten ist. Wenn jedoch eine Behauptung ausgesprochen wird, verwendet man das unbestimmte Fürwort — ,einer". Doch stets findet sich das Festhalten an der Metapher vom steinernen Pferd (30063, 1; 34067, 2 u. a.). Unter den Varianten mit anonymen Reitern ist in dieser Hinsicht das folgende Lied am interessantesten, das mit den Anfangszeilen beginnt:

Viens atjāja sirmu zirgu

Akmins segli mugurä.
Einer ritt her auf einem grauen Pferd, das einen steinernen Sattel auf dem Rücken trug.

Der Reiter ist unbekannt. Das Pferd ist grau geworden, und ein steinernes Pferd kommt nicht mehr vor. Aber die alte Vorstellung vom Reiter auf steinernem Pferd hat dennoch ihre Spuren hinterlassen. Das graue Pferd hat ,einen steinernen Sattel auf dem Rücken". Wenn man sich unter dem Eindruck dieser poetischen Sprache äußern wollte, könnte man sagen, daß der Stein mit steinerner Schwere auf dem Komplex dieser Vorstellungen ruht.

Diese schwer verständlichen und widersprüchlichen Texte zeigen bei näherer Prüfung, daß sie verständlich werden, wenn man von Ūsiņ̌s als einem Himmelsgott ausgeht.

Zum vorhin Gesagten sind zwei Bemerkungen zu machen. Erstens, 
worauf schon im Lauf der Erörterung hingewiesen wurde, darf man nicht vergessen, daB wir es mit in poetischer Form ausgedrückten Vorstellungen zu tun haben. Und daß unter diesen Texten Dichtung anzutreffen ist, die sich als Resultat spielerischer Phantasie entwickelt hat, unterliegt keinem Zweifel. Hier nur ein Beispiel $(34067,1)^{1}$.

Das Pferdchen ist zu einem silbernen geworden. Aber über das ,,steinerne Männerchen" könnte man sich vielleicht freuen, denn das stimmt mit dem oben behaupteten zähen Festhalten an der Vorstellung von Stein in diesen Texten irgendwie äberein. So könnte es scheinen. Die zweite Hälfte des Liedes zeigt jedoch, daß dieser Text nicht zu unseren Texten gehört. Das Zählen der Blätter und noch mehr das Messen des Wuchses sind völlig fremde Vorstellungen ${ }^{2}$. Wir können uns zwar freuen über diesen von der Volksdichtung beeinflußten Poeten, aber sein Gedicht gehört nur ihm selbst.

Hier ist noch eine zweite Bemerkung zu machen. Die Folge der behandelten Texte könnte den Eindruck entstehen lassen, daß sie auf eine in ihnen feststellbare chronologische Entwicklung der Vorstellungen hinweisen soll. Diese Folgerung ist falsch. Die Reihenfolge der Textgruppen wird von der Notwendigkeit bestimmt, das Problem überschaubar darzustellen. Sicherlich sind die Veränderungen der Vorstellungen, wie sie sich in den Texten spiegeln, in historischer Zeit vor sich gegangen. Doch ist es nicht möglich, innerhalb dieser bestimmte Grenzen zu ziehen. Man kann nicht nur annehmen, sondern mit großer Sicherheit sagen, daß verschiedene Vorstellungen nebeneinander bestanden haben.

5.2.3. Nach dieser Erörterung ist es jetzt an der Zeit zu fragen, ob die Quellen noch eine weitere Begründung dafür bieten, daß Ūsiňš ein Himmelsgott ist. Hier ist zuerst auf Lied 45 hinzuweisen:

45. Üsiňs brauca niedru tiltu,

Deviniem lumeliem;

Nij tam lüza niedru tilts,

Nij tam slīka kumelingš. A53
Usiñ̌s fuhr auf der Schilfrohrbrücke mit neun Rossen dim.; weder brach die Schilfrohrbrücke, noch ertrank ihm das RoG dim.

Der Text ist eigentümlich und unterscheidet sich bedeutend von all dem, was wir über Ūsinš gehört haben. Vor allem ist dieser ein Fahrer. Als Fahrer erscheint er allerdings nur in diesem Text. Seltsam ist auch die Stelle, über die er fährt, nämlich über eine Schilfrohrbrücke. Auch die Brücke selbst ist merkwürdig, weil sie nicht bricht, wenn man über sie fährt, wie man es erwarten könnte. Das einzige, was uns an bekannten Vorstellungen ge-

x Ein Teil der Texte wurde aus diesem Grunde nicht näher betrachtet; sie sind in dem am Schluß dieser Arbeit beigefügten Register zu finden. Cf. S. 166.

2 Der Dichter hat offenbar gemeint, wenn man vom Inhalt des Liedes ausgeht, daß der steinerne Mann ausmißt, wie lang das Gras gewachsen ist. Das bestätigt erneut, daß der Text eine freie Schöpfung irgendeines Dichters ist. 
blieben ist, sind 9 Pferde. Es sind Umstände, die an der Echtheit des Textes zweifeln lassen. Und hier können nur zwei Erklärungen dienen: entweder ist der Text eine Frucht reiner Phantasie, oder er bezieht sich a uf ein anderes Wesen. Echte Fahrer sind die Himmelsgötter Saule, Pērkons und vor allem Dievs. Daß die beiden letzteren über eine Brücke fahren, ist vielfach beschrieben worden ${ }^{1}$. Zum Verständnis dieses Usinšs-Liedes hat ein anderer Text eine besondere Bedeutung. Es ist das Lied 32931, das in 35 verschiedenen Varianten bekannt ist, und das inhaltlich und zum Teil auch im Ausdruck unserem Lied 45 entspricht 2 :

Dievinš brauca niedres tittu

Äbolainu kumelinu;

Tric tiltiňs, šlıind iemaukti,

Dreb Dievina kumelinšs. 32931
Gott dim. fuhr auf der Schilfrohr. brücke mit einem Apfelschimmel; es dröhnt die Brücke dim., es klingen die Zügel, es zittert das Rößlein des Dievs dim.

Auch in diesem Text treffen wir auf eine Schilfrohrbrücke, aber der Reiter ist Dievs. Von allen $35 \mathrm{~V}$. ist nur in 6 davon die Rede, daß über eine Schilfrohrbrücke gefahren wird. In überwiegender Mehrheit - in 18 Texten ist die Brücke aus Stein: zvirgzdu 'Grant', olu 'Kiesel' usw. Außer diesen 6 Fällen besteht die Brücke aus Kupfer, in einem Fall aus Sternen, und in 4 Fällen wird das Material nicht erwähnt. Damit kehren wir wieder zurück zu dem so unablässig bezeugten Material aus Stein. Zur Gruppe der Ausdrucksmittel in Metaphern gehören eigentlich auch die Vorstellungen der kupfernen und der Sternenbrücke. Mit diesen Hilfsmitteln an Metaphern wollte man zum Ausdruck bringen, daß es sich bei der Brücke um etwas besonderes handelt, ebenso wie beim darüber Reitenden oder Fahrenden.

Noch mehr Klarheit bringen die in den Texten erwähnten Reiter. Von allen 35 Texten handelt es sich $26 \mathrm{mal}$ beim Fahrer um Dievs, $8 \mathrm{mal}$ um Jānis ${ }^{3}$, und nur einmal ist ein völlig fremdes Wesen Jupis* erwähnt, in der Volkssprache die Bezeichnung für den Teufel. Diese Übersicht zeigt, daß der eigentliche Reiter über die Brücke vorwiegend Dievs ist, über die Schilfrohrbrücke reitet er zudem als einziger. Daß auch Jānis in diesen Texten erscheint, ist verständlich, da er in den verschiedensten Zusammenhängen den Himmelsgöttern angeglichen ist und an ihre Stelle tritt, insbesondere an die von Saule. Unser einziges UsinššLied, in dem eine Schilfrohrbrücke vorkommt, ist als einzige Variante der Gruppe dieser 35 Lieder anzusehen. Es zeigt sich im Lichte der übrigen Texte dieser Gruppe, daß

${ }^{1}$ Cf. Biezais 1961, 97, und 1972, $164 \mathrm{ss}$.

2 Die Varianten dieses Liedes 32931 habe ich in anderem Zusammenhang erörtert, cf. Biezais 1972, $164 \mathrm{ss}, 388$.

$s$ Über die Bedeutung von Jānis im lettischen religiösen Synkretismus habe ich mich bereits ausführlich, $1972,330 \mathrm{ss}$, geäußert.

4 Die Variante mit Jupis $(32931,9)$ habe ich, 1972, 166, gesondert behandelt. 
dieser Text zwar vereinzelt auftritt, aber auch, daß nichts im Wege steht, ihn in die Liedergruppe über die Himmelsgötter einzureihen. Ich glaube trotzdem nicht, daß dieser einzige derartige Text es erlaubt, irgendwelche sicheren Schlüsse zu ziehen. Nur soviel ist sicher, daß weder die Vorstellung vom Fahren noch von der Brücke sonst mit Üsiņš zu verbinden sind. Beide Vorstellungen sind im Zusammenhang mit Dievs und Përkons verbreitet. Das einzige, was man hier dazu sagen könnte und was der Text bezeugt, wäre, daß die Verbindung von Ūsiņš mit Dievs und Pērkons zu einem Komplex nichts Ungewöhnliches gewesen ist.

Es scheint, daß auch das schon früher behandelte Lied 50, in dem ausgesagt wird, daß Ūsiņš ,,rollte herbei" , auf dieselbe Verbindung mit dem Komplex der Himmelsgötter hinweist. Auch in einem Text unserer Gruppe von 26 Liedern mit unbekanntem Reiter ist davon die Rede, daß einer, ,hergedonnert" $\mathrm{kam}(34067,2)$. Rollen, d. h. rollend ankommen kann nur ein runder Gegenstand. Das ist schwer zu vereinbaren mit dem Reiter Ūsiṇš. Aber diese Vorstellung könnte in die Welt der himmlischen Wesen hineingehören. Es ist bezeichnend, daß auch der hl. Martin ,herdonnert" und „,davondonnert" (33242) und die hl. Katharina ,hinwegrollt" (33242, 1). Es besteht kein Zweifel, daß weder der hl. Martin noch die hl. Katharina die ursprünglichen ,Roller" und „Donnerer" sind. Ebenso konnte auch Úsinšs zum „Roller" geworden sein, wahrscheinlich unter dem Einfluß der Himmelsgötter. Aber ebenso gut könnte es sich um einen dichterischen Ausdruck handeln.

Besondere Aufmerksamkeit haben drei Texte verdient, die bereits seinerzeit von Auniņš veröffentlicht worden sind $(37,38)$ :

37. Ūsinam divi dèli, Sarkanām galvinām; Vienu süta piegulı̄, Otr' ar arklu tïrumä.

a. A50: 1-2: $\bar{U} \sin s ̌$ savus divus dētus, Darbinam radināja; 4: arti.

38. Ũsinam bij divi dẽli, Abi vienu vecuminu; Neredzēja, kad tie dzima, Tik redzëja staigājot: Prāvākais, kad strädāju, Mazakais, kad gulēju.
Ūsinš hat zwei Söhne

Mit roten Häuptern dim.:

Den einen schickt or auf die Nachthütung,

Den anderen mit dem Pfluge auf's Feld. A41

Ūsinš gewöhnt seine zwei Söhne an die Arbeit dim.; 4: um zu pflügen.

Ũsin̆ hatte zwei Söhne,

Beide von Einem Alter;

Man hat nicht gesehen, wann sie geboren wurden,

Mat hat sie nur wandern sehen:

Der Größere, wenn ich arbeitete, Der Kleinere, wenn ich schlief. A42

Im dritten Text (37a) gibt es nur geringe sprachliche Unterschiede. Der Hinweis darauf, daß Ūsiņš zwei Söhne hat, ist in anderen Texten nicht zu 
finden. Von diesen Söhnen ist bekannt, daß sie gleich alt sind, aber niemand gesehen habe, ,wann sie geboren wurden", sie werden jedoch sichtbar, wenn sie wandeln. Weiter ist gesagt, daß der eine größer ist und erscheint, während man arbeitet, also am Tage, der andere jedoch ist sichtbar, wenn man schläft, d. h. nachts. Damit stimmt auch eine andere Aussage über ihre Verschiedenheit überein, daß nämlich der eine mit dem Pflug über den Acker geht, d. h. er arbeitet am Tage, während der andere zur Nachthütung geht, d. h. er arbeitet nachts. Ein gemeinsames Kennzeichen beider Söhne ist, daß sie beide rote $K o ̈ p f e ~ h a b e n$.

Wer sind diese zwei Söhne von Ūsiņšr? Auf diese Frage hat bereits Auniṇš bei der Textveröffentlichung versucht zu antworten: „Diese beiden Söhne UhBings sind ohne Zweifel das Abendrot und das Morgenrot." Aber auch über die im zweiten Text genannten zwei Söhne hat er keinen $Z_{\text {weifel: }}$ „,Der Sinn dieses Liedes ist wiederum leicht zu erraten. Diese beiden Söhne Uhßings, die man nur stets wandern sieht, und zwar den einen am Tage und den anderen in der Nacht, können nichts Anderes als die Sonne und der Mond sein. "2 Daraus kann man entnehmen, daß Auniņš zwei verschiedene Traditionen annimmt, die die Söhne von Ūsinsš mit zwei verschiedenen Natursubstraten verbinden. Es ist bezeichnend, daß Schröder, der sonst das Material und größtenteils auch die Ansicht von Auniņš über Ūsiņš allgemein übernommen hat, über die Söhne von Usiņš jedoch schweigt. Und das ist verständlich, denn Schröder identifiziert Usiňš mit der Frühlingssonne. Er hatte sich schon früher eingehend mit der Frage der Sonnentöchter beschäftigt und unter Berufung auf lettisches Material festgestellt, daß es sich um die Morgenröte handele. Mit dieser seiner Ansicht war die Vorstellung von Söhnen nicht vereinbar. Es scheint, daß er deshalb gezwungen war, das Problem der Söhne des Usiṇ̆s mit Schweigen zu übergehen.

In Wirklichkeit ist das Material über die beiden Söhne viel umfangreicher. Der Schwerpunkt liegt tatsächlich in diesem Falle bei der Zahl zwei. Zudem verbinden sich damit mehrere Vorstellungen: zwei Söhne, zwei Pferde, zwei Kerzen usw ${ }^{3}$. Deren Eigentümer sind verschiedene Himmelsgötter: Mēness, Diers, Saule, Pērkons ${ }^{4}$. Nach einer eingehenden umfangreicheren Analyse der Textgruppe habe ich mich schon früher über diese Erscheinung geäußert und erkannt, daß hinter diesen Vorstellungen als Natursubstrat der Morgen- und der Abendstern stehen ${ }^{5}$. Aber das bedeutet nicht, daß

1 Auniņš 1881, 32.

2 Ib. Diese Ansicht hat er 25 Jahre später noch einmal bezeugt (cf. Auniņ̌̌, 1905,

21). Danach hat auch Lautenbachs, 1896, 142, diese Auffassung übernommen.

3 Näheres darüber bei Biezais 1972,455 ss.

4 Cf. ib. 33,457 ss u. a.

5 Ib. 467 .

\section{4}


man nun mit mathematischer Genauigkeit andere Natursubstrate ausschließen könnte. Variationen sind hier schon deshalb möglich, weil die Vorstellung von Söhnen von der sozialen Umwelt bestimmt wird, in der sie sich gebildet hat ${ }^{1}$. Diese Bemerkung ändert grundsätzlich nichts an der Erkenntnis, daß sich hinter den Söhnen der Himmelsgötter der Morgenund Abendstern verbergen. Aus diesen haben sich die mythischen Gestalten der Dieva dēli 'Gottessöhne' entwickelt mit ihren besonderen Funktionen und ihrer Stellung in der Religion der alten Letten. Es handelt sich um einen der ältesten Züge dieser Religion, die diese mit altindischen religiösen Vorstellungen, den divō napātā, den griechischen $\Delta$ iò « xoũpot, verbindet ${ }^{2}$.

Wenn wir uns unseren beiden Texten (37) wieder zuwenden, muß man feststellen, daß die hier erwähnten zwei Söhne, von denen der eine am Abend erscheint und die Pferde zur Nachthütung reitet, während der andere morgens auf dem Felde pflügt, mit dem vorhin Gesagten übereinstimmen. Das wird auch durch die Variante bestätigt, in der Ūsiņš seine Söhne in die Arbeit einführt, nämlich nachts die Pferde zu hüten und am Tage den Acker zu bestellen.

In dieselbe Richtung zielt auch die Behauptung, daß die beiden rote Köpfe hätten. Auch der dritte Text (38) bestätigt das gleiche mit den Aussagen, daß beide Söhne in einem Alter seien und daß keiner ihre Geburt gesehen habe, sondern daß sie nur zu sehen seien, wenn sie wandelten. Dieser Text kann Bedenken hervorrufen, da zu den üblichen 4 Zeilen noch 2 Zeilen mit der Erklärung angefügt sind, daß von diesen wandelnden Söhnen des Ūsiņš der eine größer, der andere kleiner sei, daß der größere wandele, „wenn ich arbeite", d. h. am Tage, der kleinere jedoch, ,wenn ich schlief", d. h. in der Nacht. Das läßt vermuten, wie schon Auniņš sagte, daß mit diesen Söhnen eindeutig Sonne und Mond gemeint sind. Doch ist zu beachten, daß diese beiden letzten Zeilen einen Zusatz zum Text darstellen. Sie zeigen, daß der Schöpfer des Textes schon damals in derselben Lage war wie wir heute - in Unklarheit, was mit diesen beiden Söhnen gemeint sei. Und er hat dann für sich Klarheit geschaffen, indem er sich entsprechend dem Zusammenhang für den größeren tags und den kleineren nachts entschied. Diese erklärenden zusätzlichen Zeilen stellen eine spätere, vernunftmäßige Begründung dar.

Dem Text 37 hat sich auch Endzelīns zugewandt und ihn benutzt, um seine Ansicht, daß Ūsinšs in diesem Text als Hausgeist anzusehen sei, zu bekräftigen $^{3}$. Welcher Zusammenhang zwischen diesem Hausgeist und

1 Darüber habe ich mich 1972,67 ss, 115 ss, 183 ss, ausführlicher geäußert; auch 1961,69 ss.

2 Cf. Biezais 1972, $467 \mathrm{~s}$.

3 Cf. Endzelins 1903, 26; dazu Auniņš 1905, 27. 
den zwei Söhnen mit roten Köpfen bestehen soll, hat er nicht erklärt. Er hat auch die Echtheit des Textes 38 bestritten, da dieser ihm ,sowohl in bezug auf den Rhythmus als auch auf den Inhalt Anstoß zu erregen schien", aber dazu haben wir uns schon früher geäußert. Bekanntlich hat er sich geirrt.

Eine Vertiefung in diese Texte zeigt noch deutlicher, daß Ūsiňš zur Familie der Himmelsgötter gehört. Hier könnte man einwenden, daß diese Vorstellungen sich unter dem direkten Einfluß anderer Himmelsgötter gebildet haben. Man kann die Frage weder bejahen, noch verneinen. Doch das ändert nichts an der tatsächlichen Situation, da die Funktionen der himmlischen Götter sich vielfach überschneiden und zusammenfallen. Dadurch ändert sich ihr Wesen als Himmelsgötter nicht. Vielleicht ist eine negative Aussage hier am bezeichnendsten: der hl. Georg nämlich, der so tief in die Funktionen des Usinšs eingedrungen ist, wurde im Komplex dieser Vorstellungen nicht mit Söhnen bedacht.

Hier sind noch ein paar Texte zu erwähnen, die bereits von Auniņš ausgewertet worden sind, um die Identität von Üsinš mit der Sonne zu begründen. Es sind unsere Lieder 11 und 12: beide wurden schon früher abgedruckt. Nun haben sich die Forscher auf die darin vorkommenden Vorstellungen berufen, daß ,Ūsinšs richte sich hoch auf “ (11) und daß „Üsiňš tanzte, Usiņš sprang“ (12). Auniņš sagt dazu, unter Berufung auf Bielenstein, letzterer stelle, die Hypothese auf, daß mit dem stāvu slaistities nicht angedeutet sei, das Roß des UhBing bäume sich, sondern Uhßing selbst richte sich hoch auf. Hiemit werde das höhere und höhere Aufsteigen der Frühlingssonne am Firmament bezeichnet. Von dieser hochaufsteigenden Frühlingssonne wird nun gebeten, daß sie auch in den Stall und in das Haus ihren Segen spende, auch auf den EBtisch, an dem der Uhßing den Ehrenplatz einnehmen soll cf. Nr. 1 .

In Nr. [12] springt und tanzt Uhßing hinter dem Pferdestall und wird gebeten in den Pferdegarten (die Pferdekoppel) hineinzuspringen. Man beachte bei diesem Liede, daß lēkt der constante Terminus für das Aufgehen, Aufsteigen der Sonne ist." 1

Diese Ansicht von Auniňš hat Schröder übernommen. Indem er sich auf einige dieser Texte von Auniņš stützte und sie mit einigen mehr oder weniger ähnlichen Texten näher verglich, in denen Saule genannt wird, äußerte er sich: , [...] es liegen eine ganze Reihe von Anzeichen vor, charakteristische Züge im Wesen des Gottes [Ūsinšs], welche in ihm, wie ich glaube, unzweifelhaft einen alten Sonnengott erkennen lassen, der sich aufs beste in die bunte Reihe der uns bereits bekannten altarischen Sonnengottheiten einfügt ${ }^{\varsigma / 2}$.

1 Auniņš 1881, 25 s; cf. auch 1905, 18. Doch fehlt bei Auniņš der Hinweis, wo Bielenstein diese Ansichten geäußert hat.

2 Schröder 2, 51 . 
Und einige Seiten weiter kommt er zu der sicheren Überzeugung: ,Daß Uhsing wirklich die Sonne ist, ergibt sich endlich auch noch daraus, daß in ganz einander ähnlichen, wie Varianten aussehenden Liedern einmal Uhsing und ein anderes Mal 'die Sonne' steht."1

Zunächst muß man zu dem letzten Argument Schröders sagen, daß wir in dieser Arbeit mehrfach auf die Erscheinung gestoßen sind, daß in verschiedenen Varianten ein und desselben Liedes neben Üsinšs verschiedene Wesen auftreten. Aber wir haben auch daraus gelernt, daß das kein Grund ist, Ūsiņš mit diesen Wesen zu identifizieren, geschweige denn, daraus Folgerungen über das ursprüngliche Wesen des Ūsinšs zu ziehen.

In noch größerem Maße als Saule sorgt Dievs für die Nachthüter². Jedenfalls bietet der von Schröder benutzte Text über Saule keine ausreichende Grundlage, um Usiņš mit dieser zu identifizieren. In Wirklichkeit ist sein Argument ein zusätzliches Argument zu den Texten über Ūsiņ̌s, der hinter dem Stall steil aufsteigt, springt und tanzt. Es stimmt, daß Saule in den lettischen Traditionen ,,springt" und, ,tanzt" ${ }^{* 3}$. Daher ist auch das Bestreben zu verstehen, Usinšs, der in ähnlichen Situationen erscheint, mit Saule zu identifizieren.

Hier ist zunächst an die Übersetzung der Worte $\bar{U} \operatorname{siņš~stāvu~slaistījāas~}$ durch Auniņš (sowie Bielenstein) und Schröder zu denken: ,Uhßing richtete sich hoch auf ${ }^{\leftarrow}$. Auniņš hat wiederholt, unter Berufung auf Bielenstein, bemerkt, daß die Worte stāvu slaistījōs nicht so zu verstehen seien, als ob „,das Roß des Uhsing" sich „bäume" [...], ,sondern UhBing selbst richte sich hoch auf" drucks angebracht ist. Vor allem kennt man das Verb slaistïties, in der 3. P. Singl. Impf. slaistījās, dessen Nebenbedeutung ,sich bäumen" ist, auch in der Bedeutung: ,faul bei der Arbeit sein [...], sich ohne Arbeit herumtreiben" ${ }^{\prime 6}$. Davon ist das Substantiv slaists 'ein fauler Schlingel, ein Müßiggänger, Herumtreiber, Bummler' abgeleitet". Der erste Teil der Redewendung enthält das Adverb stävu und bedeutet soviel wie ,stehend, aufrecht ${ }^{\text {‘ } 8}$. Das ist so zu verstehen, daß der Zustand sich vom Sitzen oder Liegen unter-

1 Ib. 55. Hier beruft er sich auf den bei Aunį̣š 1881, 27 erwähnten Text, der in Wirklichkeit jedoch von Sprogis, 285 , übernommen ist.

2 Cf. Biezais 1961, 147 ss.

3 Cf. Biezais 1972, 322 ss; auch Edsman 1958, 21 ss.

4 Endzelins 1903, 26, hat diesen Text abgelehnt, weil er jüngeren Datums sei, in Wirklichkeit aber wohl deshalb, weil er nicht mit seiner Hypothese vom Hausgeist übereinstimmte.

5 Auniņš 1881, 25; cf. auch 1905, 18 .

( ME 3, 914.

$7 \mathrm{Ib}$.

8 Ib. 1055. Cf. auch Grīnberga, Elza 292. 
scheidet. Diesen Sinn hat das Wort auch in der heutigen Sprache. Aus dieser semantischen Bedeutung ergibt sich auch der Sinn des ganzen Ausdrucks - es soll nämlich heißen, daß sich hinter dem Stall jemand ohne Arbeit und faulenzend aufgehalten hat. Sicherlich schließt das nicht die Möglichkeit aus, die Wortgruppe stāvus slaistījās in anderem Kontext so zu verstehen, wie es der heutigen Sprache entspricht: ,stieg auf“", ,, hob sich empor". Jedenfalls ist das richtige Verständnis dieser Wortgruppe vom Kontext abhängig. Wie sie richtig zu interpretieren ist, zeigt die zweite Hälfte unseres Textes:

$E j, \bar{U} \sin$, stallïtī, Baro labus kumelinus.

Geh, Usin, in den Stall dim., füttere die guten Rößlein.

Daß der Ausdruck in der ersten Hälfte des Textes den faul Herumstehenden meint, wie wir eben ausführten, zeigt die Aufforderung zu aktiver Tätigkeit in der zweiten Hälfte: Usinšs möge in den Stall gehen und die Pferde füttern. Das heißt, daß es keinen Grund gibt, den faul Herumstehenden für Saule zu halten.

Auniņ hat ferner betont, daß nicht das Pferd von Usiņšs sich bäume, sondern dieser selbst. Das wurde dann mit dem Sinn des Wortes slaistities 'sich hoch aufrichten' kombiniert und verhalf dazu, Ūsiņš mit der Frühlingssonne zu identifizieren. Der Ausdruck slaistīties zwingt nicht dazu, eine Grenze zwischen Ūsiņš und seinem Pferd zu ziehen. Hier kann man auf den gleichen Ausdruck verweisen, wie er auch in einer Daina zu finden ist: ,steil stieg das Rößlein in die Höhe“" (11046). Er ist eindeutig und bezieht sich nur auf das Pferd. Andererseits ist ebenso klar, daß es sich in unseren Texten 11 und 12 um Usinš handelt. Aber das ist zu verstehen, denn auf dem Wege der Kontamination konnte an die Stelle des in anderen Texten erwähnten Pferdes in unseren Texten der Pferdepatron treten.

Die in Varianten festzustellende Aufforderung, U̦siņš möge nicht hinter dem Stall faulenzen, sondern in die Stube kommen und sich ans Ende des Tisches setzen, oder, in anderen Varianten, er möge in den Stall oder auf die Pferdekoppel gehen, wollen Auniņš und andere so verstehen, daß damit die Frühlingssonne aufgefordert werde, mit ihrer wohltuenden Wirkung auch in den Stall und in die Stube zu kommen. Es stimmt, daß Saule die Spenderin der Fruchtbarkeit der Felder und überhaupt die Fördererin des Wohlergehens ist. Aber es ist bedeutsam, daß sie im ganzen großen Folklorematerial als Göttin niemals aufgefordert wird, in die Stube oder in den Stall zu kommen. Ihr Platz ist gewöhnlich draußen'1. Hier genügt der Hinweis, daß Usinšš, der am Rande des Hofes sitzt oder steht, aufgefordert wird, in die Stube zu kommen (1). Dabei besteht weder ein direkter noch ein

1 Cf. Biezais 1972, 803 ss. 
indirekter Zusammenhang mit Saule. Ūsinšs, der zu den Göttern gehört, die das Wohlergehen des Bauers fördern, wird immerzu aufgefordert, in dessen Haus zu kommen, je nachdem, entweder in die Stube, den Stall, auf die Koppel oder auch zur Nachthütung.

Unleugbar ist der wichtigste Hinweis, den Auniņš verwendet, die Stelle im Text 12, wo „Ūsiṇš tanzte, Ūsiņš sprang“. Tanzen und Springen ist stets mit der Sonne verbunden. Auniņš betont das besonders, da es der „constante Terminus für das Aufgehen, Aufsteigen der Sonne ist". Das Verb lēkt 'springen' wird tatsächlich so gebraucht. Aber es ist nur eine der vielen semantischen Bedeutungen dieses Wortes ${ }^{1}$. Es genügt, sich nur daran zu erinnern, daß man auch beim Erscheinen des Mondes ebenso wie von der Sonne sagt: mèness lec 'der Mond geht auf'. Daher kann man die semantische Bedeutung dieses Wortes nicht nur auf das Aufgehen der Sonne beschränken. Man kann den Gedanken weiterführen und bei einer weiteren, abgeleiteten Form dieses Wortes verweilen: lēkāt 'wiederholt springen, hüpfen'2. Zuweilen verbindet sich das von diesem Verb abgeleitete Nomen mit einem freudigen Erlebnis. Man sagt gewöhnlich: viņš lekkā aiz prieka 'er springt vor Freude'.

Hier sei einer unserer Texte in Erinnerung gebracht $(67)^{3}$.

Ūsinšs der hinter dem Stall ,,richtete sich hoch auf", wird diesmal weder in den Stall noch in die Stube gebeten, sondern soll als Pferdehirte mit zur Nachthütung kommen. Das Bild ist uns von der vorangegangenen Erörterung wohlbekannt. Schwierig ist es jedoch, die nächtliche Hütung mit der von Schröder vertretenen Frühlingssonne zu vereinbaren. Dieser Text ist gegen Schröder nur zusammen mit anderen Hinweisen zu verwenden. Er ist für sich allein nicht sicher und weist mehrere Anzeichen auf, die an seiner Echtheit zweifeln lassen. Er ist eher eine Kombination zweier bekannter Liedelemente.

Im Rückblick auf die wenigen Texte über Ûsiņ̌s, der aufsteigt oder hinter dem Stall springt und tanzt, muß man sagen, daß sie keine genügende Grundlage für eine Identifizierung mit der Sonne bieten. Damit wird in diese Texte zuviel hineingelegt. Doch ist das begreiflich. Erstens war Auniņ̌ kein so umfangreiches lettisches Sprach- und Dainamaterial zugänglich, wie es heute zur Verfügung steht, geschweige denn Schröder. Überhaupt war damals mit einer ernsthafteren Erforschung des lettischen Wortschatzes in semantischer Hinsicht noch gar nicht begonnen worden. Zudem darf man nicht vergessen, daß Mannhardt bereits im Jahre 1875 seine damals so populäre Studie über die lettischen Sonnenmythen veröffentlicht hatte.

1 ME 2, 458, verzeichnet 8 verschiedene. Bedeutungen.

Cf. ib. 456 .

3 Cf. S. 83 . 
Unter deren Einfluß fiel es Schröder leicht, in den hier erwähnten Texten die Identität von. Úsiňš und Saule festzustellen ${ }^{1}$. Bei nüchterner Betrachtung dieser Texte muß man sagen, daß Ūsinš erstens in seiner gewöhnlichen göttlichen Funktion als Pferdepatron erscheint, und zweitens, daß sich mit ihm ein Teil derselben Vorstellungen wie mit den anderen Himmelsgöttern verbindet, bzw. daß er zu ihrer Familie gehört. Dennoch tritt auch in diesen Texten das eigentliche Wesen von Usiņš nicht weiter zutage.

In dieser Übersicht haben wir mehrere Textgruppen erörtert, die sich bedeutend von jenen unterscheiden, wo mehrere christliche Heilige in Funktionen von Ūsiņš auftreten. In allen diesen untersuchten Gruppen kann man in der Tätigkeit von Ūsinšs, seinen Attributen und Eigenschaften Züge erblicken, die ihn mit vorchristlichen Vorstellungen verbinden. Mehrere dieser Eigenschaften zeigen seine Zugehörigkeit zu den Himmelsgöttern.

5.3. Noch größere Klarheit über das Wesen von Ūsinš können wir erhalten, wenn wir uns nochmals seinem Kult zuwenden und diesen im Licht der bisher gewonnenen Erkenntnisse betrachten. Bei der Erörterung der Texte über den Kult des Usiņš sind wir mehrfach auf schwer erklärliche Erscheinungen gestoßen. Deshalb mußten auch mehrere Fragen offen bleiben. Wir können jetzt versuchen, sie zu beantworten, soweit das möglich ist, oder sie wenigstens einer Lösung näherzubringen.

5.3.1. Die erste Frage bezieht sich auf das Opfer und das Opfermahl. Ohne daß wir hier nochmals den Ablauf darstellen, läßt sich die zentrale Bedeutung, die gerade das Hahnenopfer einnimmt, nicht übersehen. Das Hahnenopfer verbindet man gewöhnlich mit Lichtgottheiten, worauf bereits Scheftelowitz wiederholt hingewiesen hat ${ }^{2}$. Doch nimmt es bei den

1 Jedenfalls war es größtenteils eine Zeiterscheinung, als man unter dem Eindruck. der sogenannten Sonnenmythologie den Einfluß der Sonne auf die verschiedenen religiösen Gestalten zu erkennen meinte. Das möge ein Beispiel aus derselben Zeit illustrieren, in dem das Fest des hl. Georg mit einem Sonnenfest identifiziert wird, Volksdichtung 21 s: ,Wer am Sanct-Georgstage auf der sonnigen Hochfläche des herrlichen Awala-Kegels südlich von Belgrad den Tanz der aus allen Dörfern der Umgegend herbeigekommenen serbischen Landleute angesehen, der kann nicht zweifeln, daß diese Feier nichts ist als die nur durch den Kalendernamen des Tages mit dem Chriftentum in Verbindung gebrachte Fortsetzung des dem siegenden Sonnengott auf jener beherrschenden Höhe dargebrachten Festreigens." Cf. ib. 34 über die Identität des hl. Georg und der siegenden Sonne.

${ }^{2}$ Scheftelowitz 1914, $55 \mathrm{~s}$ : ,Der Hahn ist als Verkünder des Lichtes von den alten Persern hochgeehrt und von manchen anderen Völkern mit dem Sonnengott in Beziehung gebracht und für heilig gehalten worden. Die nichtarischen indischen Stämme opfern der Sonne einen weißen Hahn. Im indischen Opferritual wird beim Aśvamedha den Lichtgottheiten, wie Savitar, Agni und Anumati ein Huhn dargebracht. So war der Hahn in Griechenland dem Helios geweiht, er war den Galliem ein heiliges Tier, das man nicht essen durfte und dessen Bild als Heereszeichen diente. Ebenso war er den 
europäischen Völkern unter den Formen des christlichen Synkretismus einen bedeutenden Platz als Apotropäum ein ${ }^{1}$. Im Fruchtbarkeitskult ist es mit dem hl. Georg und anderen in unseren Texten erwähnten Heiligen verknüpft². Daher ist das Hahnenopfer allein kein Beweis für den vorchristlichen Charakter des Ūsinšs. Es handelt sich dabei um sehr alte religiöse Traditionen, die schon besonders bei den Arabern und Semiten sowie bei anderen Völkern bekannt waren ${ }^{3}$. Sie lebten im Christentum weiter. Dadurch konnten sie auch zu den Letten gelangen. Objektiv gesehen, läßt sich nicht behaupten, daß diese Traditionen etwa den Letten in vorchristlicher Zeit unbekannt gewesen sind. In diesem Fall geht es um das Hauptanliegen des bäuerlichen Lebens, wenn es sich darum handelt, die Fruchtbarkeit der Felder zu sichern und sich vor bösen und schädlichen Kräften zu schützen. Das religiöse Erlebnis, das damit verbunden ist oder, richtiger, die Motivierung bildet, ist die Grunderfahrung. Es läßt sich schwer annehmen, daß sich solch ein Opfer erst zur Zeit des christlichen Synkretismus bei den Letten eingebürgert haben soll, wenn es schon lange vor dem Christentum bei anderen Völkern weit verbreitet war. Unter dem Einfluß der christlichen Kirche verband es sich zwar mit den Heiligen, die aber selbst vielfach Substitute vorchristlicher Götter waren. Mangels historischer Quellen ist es nicht möglich, über diese prinzipielle Behauptung hinauszugehen.

5.3.2. Anders verhält es sich mit dem schon früher behandelten Mahl der Nachthüter. Auch die Tradition Westeuropas kennt Mahle, die mit den Festen des christlichen Kalenders verknüpft sind. Doch in dem sogenannten Mahl der Nachthüter sind mehrere Momente enthalten, die es in einem besonderen Licht erscheinen lassen. Zunächst die Zeit des Mahles: es 1 indet am späten Abend statt, in Wirklichkeit dann, wenn es schon dunkel gegeworden ist. Seine Teilnehmer sind besonders ausgesucht, es sind die Nachthüter, alles Männer. Es handelt sich um Mitglieder mehrerer Hausgemeinschaften. Das wichtigste Kennzeichen ist, daß die zum Mahl benötigten Dinge von den Teilnehmern zusammengelegt werden. Jedenfalls handelt es sich, was nicht nur für die Letten gilt, um eine sehr alte vorchristliche Tradition ${ }^{4}$. Hierbei muß man sich auch an die eigenartige Zu-

Slaven ein heiliger Vogel. Auch bei den Germanen kommt diese Anschauung vor. Nach Ditmar von Merseburg gehörte der Hahn zu den edelsten Opfern, die auf Seeland jedes neunte Jahr den Göttern geopfert werden mußten." Cf. auch id. 1925, 75.

1 Scheftelowitz 1925, 74 s: ,Als dämonenverscheuchendes Tier ist der Hahn bei den verschiedensten Völkern angewandt worden, so bei den altpalästinensischen Juden, bei den verschiedensten Völkern Asiens, Afrikas und Europas."

2 Cf. Sartori 3, $168 \mathrm{~s}$.

${ }^{3}$ Cf. HDA 3, 1328.

4 Cf. Biezais 1972, 146 ss. 
bereitungsweise des Mahles erinnern, wie sie von Bielenstein und auch im Folklorematerial beschrieben wird, nämlich an einer Feuerstelle. Das Mahl hat einen ausgesprochen sakralen Charakter. Sowohl an der Zubereitung als auch am Mahl nimmt Ūsiņš selbst teil. Zur Bereitung des Mahles bringt er den Nachthütern Eier. Dasselbe, was vom Hahn zu sagen war, muß auch über die Verwendung von Eiern zum Opfermahl gesagt werden. Über die Rolle von Eiern in vorchristlichen Kulten, sowohl als Symbolen der kosmischen Ordnung als auch als Lebens- und Fruchtbarkeitssymbolen, ist viel geschrieben worden ${ }^{1}$. Nichts steht dem entgegen, diese vorchristliche Symbolik auch auf die von Üsiṇš mitgebrachten und fürs Mahl verbrauchten Eier zu beziehen. Doch fehlt es in den Quellen an solchen offen genannten Beweggründen. Daher kann man sich nur an die Feststellung der Tatsache halten, daß die Teilnahme von Ūsiņš bei der Bereitung des Mahles und beim Verzehr den sakralen Charakter sichert. Die Verwendung gerade von Eiern und anderen Lebensmitteln zum Mahl kann auch ökologische Ursachen haben.

Tch habe bereits früher in anderem Zusammenhang darauf hingewiesen, daß die lettischen Himmelsgötter selbst mithelfen, das während des Mahles

1 Dazu bietet HDA, 2, $595 \mathrm{~s}$, einen umfangreichen Literaturhinweis. Die Vorstellungen über die kosmische Bedeutung des Eies sind sehr verbreitet und alt: „Die Vergleichung der Eiform mit der Gestalt des Alls wird öfter hervorgehoben. Aus den beiden Hälften des Eis sind Himmel und Erde hervorgegangen. Nach der indisehen Kosmogonie wohnt Brahma lange Zeit verborgen im Urei, dann teilt er es in zwei gleiche Hälften und macht aus der einen Hälfte den Himmel, aus der andern die Erde. Nach ägyptischer Lehre erzeugt Kneph aus seinem Munde das Urei, welches man als Weltbild auffaßte. Phthas, der bei Suidas 'A $\varphi \vartheta \alpha \dot{\zeta}$, Dionysos heißt, eröffnet es, aus der einen Hälfte entsteht der Himmel, aus der andern die Erde. Nach der sidonischen

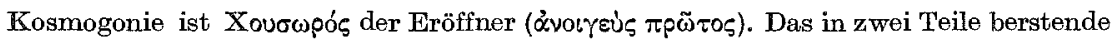
Ei bildet Himmel und Erde. Bei den Chaldäern zerschneidet Bel, der Demiurg, die Omoroka oder Omorka; die obere Hälfte ist der Himmel, die untere die Erde" (Bachofen $30 \mathrm{~s}$ ). (Cf. auch den orphischen Text bei Nilsson 1927, 51). Es ist verständlich, daß Bachofen eine kosmische Philosophie entwickeln konnte: "So ist also das Ei in

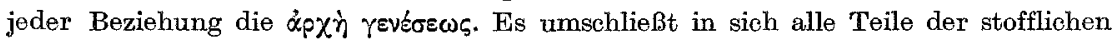
Welt, Himmel und Erde, Licht und Finsternis, die männliche und die weibliche Naturpotenz, den Strom des Werdens und des Vergehens, den Keim aller tellurischen Organismen, der höhern und der niedern Schöpfung, und die ganze Götterwelt, die, stofflichen Ursprungs wie alles tellurische Leben, mit Menschen, Tieren, Pflanzen eine

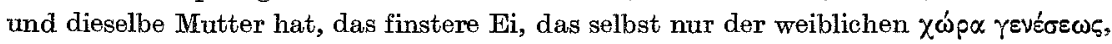
in welcher Isis und Osiris schon vor der Geburt liebend sich umfangen, entsprechendes Abbild ist." (ib. 33). Ungeachtet dessen, daß Nilsson, 1908, 543, Bachofens kosmische Auffassung für richtig hält, hat er sie dennoch, so scheint es, von seinem Standpunkt als augesprochener Rationalist als mystisch angesehen. Ähnliche Vorstellungen aus Afrika hat Sicard, $905 \mathrm{ss}$, bemerkt.

Neben der kosmischen Symbolik verbinden sich mit dem Ei auch Fruchtbarkeits. riten, wie es das Eieropfer bei Begräbnissen schon in der Vorzeit bezeugt: Nilsson, 1908, 
genossene Getränk - Bier - zu bereiten ${ }^{1}$. Usinš bildet in dieser Hinsicht, wie wir bei der Quellenanalyse gesehen haben, keine Ausnahme. Auch er braut Bier.

Ich möchte noch einmal auf die vorhin erwähnte Bereitung des Mahles an einer Feuerstelle hinweisen und deren Bedeutung betonen. Die Textanalyse bietet Grund zu der Behauptung, daß die Feuerstelle ein sakraler Ort ist. Andererseits glaube ich nicht, daß diese Tatsache Spekulationen zuläßt, ihn mit dem Kult der Saule zu verbinden. Das mag unter anderen Umständen zutreffen, aber in unseren Quellen gibt es darüber keine Aussagen. Ebenso muß die Frage über Ort und Rolle des kultischen Liedes offen bleiben, da es vorläufig an jeglichen Forschungen auf diesem Gebiet fehlt.

Die hier dargestellten Merkmale machen deutlich, obwohl christliche Heilige mit einbezogen werden, daß sie einer anderen Welt religiöser Vorstellungen angehören, in diesem Falle der lettischen vorchristlichen Religion. Wenn man die in diesem Usinšs-Kult erkennbaren alten Züge mit dem vorhin Gesagten über Usiñš als Himmelsgott verbindet, können wir dessen Gestalt jetzt deutlicher erkennen.

5.4. Als Resultat dieser Forschungen sind mehrere Erkenntnisse zu verzeichnen, die mehr Klarheit in diese vieldiskutierten Fragen bringen.

5.4.1. Die Quellenforschung ergibt keine Bestätigung der Ansichten, nach denen der Gott Usinš in der Religion der Letten eine Entlehnung aus deutschen oder russischen Traditionen sein soll. Weder sein Name noch sein Wesen sind auf diese Weise zu erklären, da in den Traditionen dieser Völker Zeugnisse über ein Wesen, das Usiņš entsprechen würde, fehlen.

5.4.2. Es gibt keinen Grund für die Behauptung, daß Ūsinš eine Neuschöpfung sei, die in die Religion der Letten unter dem Einfluß christlicher Heiliger, insbesondere des hl. Georg, Eingang gefunden habe. Diese Ansicht bietet keine Erklärung für den Namen Usiņš, der, wie wir gesehen haben, bereits seit dem 16. Jh. in Orts- und Personennamen bezeugt ist. Es ist

544, hat drei Möglichkeiten der Auslegung solcher Opfer genannt, nämlich als mystische Lebenskraft (Bachofen), als Aphrodisiaca (Poulsen) und als Neuzeugung (Dieterich). Er selbst hat sich der letzteren Ansicht angeschlossen. Hinweise, die bei Mogk, 220, geäußert werden, kommen dem in unserer Arbeit behandelten Problem noch näher: „Wie im Leben des Ackerbauers hat auch in dem des Viehhirten das Ei ganz ähnliche Bedeutung gehabt. Bei dem ersten Austrieb wird der Hirt mit Eiern beschenkt, muss er Eier essen. Das Vieh muss über Eier schreiten, muss sie zertreten. Was aber vor allem in den Kreis des Fruchtbarkeitszaubers einschlägt, ist die Sitte, die Eierschalen auf der Weidewiese zu vergraben. Nach dem Volksglauben soll sich dann das Vieh nicht verlaufen. "Eine gute Übersicht tuber die verschiedenen Riten bietet auch Lukas 228 ss; of. auch Haberland 59; Mannhardt 1875a, 158, 291.

1 Cf. Biezais 1961, $123 \mathrm{~s}$. 
nicht glaubhaft, daß diese unter dem Einfluß des Christentums entstandene Neuschöpfungen darstellen sollen. Die Quellen bezeugen eindeutig das Gegenteil, nämlich, daß mehrere Heilige, der hl. Georg, der hl. Michael, der hl. Martin, in der Zeit, als sich der christliche Synkretismus bildete, in die Vorstellungen eingedrungen sind, die sich mit Ūsin,š verbinden. Doch waren sie niemals in der Lage, weder alle Funktionen von Ūsinš zu übernehmen, noch in alle kultischen Vorstellungen Eingang zu finden. Die christlichen Heiligen haben gerade diese Merkmale des ŨsinššKultes, die ihn mit den Himmelsgöttern verbinden, nicht erlangen können. Diese Heiligen konnten weder Söhne haben, noch konnten sie über den steinernen Himmelsberg reiten. Es ist unmöglich, da $ß$ sich diese mit Ūsinš verknüpften Vorstellungen, die mit der Lehre von den christlichen Heiligen nicht in Einklang zu bringen sind, in christlicher Zeit gebildet haben. Das wird indirekt auch dadurch bestätigt, daß diese Heiligen ohne Vorbehalt an Stelle von Ūsinš̌ als Pferdepatron treten konnten, da das ihrer christlichen Natur nicht entgegenstand.

5.4.3. Unsere Untersuchung zeigt, daß Ūsiņ̌ ein Himmelsgott ist oder - übereinstimmend mit einem von mir früher gebrauchten Ausdruck der großen himmlischen Götterfamilie der alten Letten angehört. Hierbei teilt er in typischer Weise die Eigenschaften der übrigen Glieder dieser Gemeinschaft - er ist Reiter und besitzt Pferde. Er reitet ebenso wie jene über den Himmelsberg und erscheint zu gegebener Zeit beim lettischen Bauern. Das geschieht im Frühling. Zeitweise haben Forscher ihn in Anbetracht seiner engen Beziehung zu Pferden als Pferdegott bzw. Pferdepatron angesehen. Das ist tatsächlich so, seine Fürsorge für die Pferde ist groß. Doch ist dieses Bild so übermächtig geworden, daß andere Umstände weniger beachtet wurden. Einzelne Forscher haben bereits nachgewiesen, daß die Funktionen von Ūsiñ̌ umfangreicher sind, wie das auch unsere Quellen eindrucksvoll bezeugen. Hier ist nur an jenes auffallende Moment zu erinnern, das sich in unseren Texten so oft wiederholt, daß Üsinš, über den Berg herreitend, den Bäumen Blätter und den Feldern grünes Gras bringt. Es scheint, daß gerade hierin die tiefere Bedeutung von Ūsinš und seinem Wesen als Himmelsgott sichtbar werden. Die grünen Blätter und das grüne Gras sind Metaphern für das Erscheinen des Frühlings. Sie sind ein anschaulicher Beweis, daß das Leben wiederkehrt. Man muß die völlige Abhängigkeit des Bauers vom Erwachen der Natur kennen und sich darüber klar werden. Das Frühjahr wird in den Traditionen des Bauers auch als Hungerzeit bezeichnet, worauf sich auch in unseren Texten Hinweise finden $(4,13,15,20)^{1}$. Vor dieser Not und diesem Elend konnte der Bauer nur durch

1 Es ist wiederholt darauf hingewiesen worden, welches Elend bei den Bauern im Frühjahr herrschte und welche Erleichterung die Möglichkeit bot, das Vieh im Frühjahr 
ein gutes Frühjahr mit frischem Gras bewahrt werden. Es war eine im Leben des Bauern sehr wichtige Situation. Das bezog sich nicht nur auf die Pferde. Hier sehen wir auch eine Lösung des öfters diskutierten Problems der weiteren Funktionen von Ūsiņš. Er kümmert sich selbst um Kälber und Schafe und sogar um die Aussaaten von Roggen und Hafer. Ganz recht, denn auch das gehört zu dem erwarteten guten Frühjahr. Daher gibt es, wenn man Ūsiņš als Himmelsgott betrachtet, in Wirklichkeit gar kein Problem irgendwelcher erweiterter Funktionen. Nur der Drang des Wissenschaftlers nach Systematisierung, der einen Gott einzig an seine besonderen Funktionen binden will, schafft Probleme, wo solche im Bewußtsein des Bauers im wirklichen Leben gar nicht bestehen. In diesem Zusammenhang ist daran zu erinnern, daß bereits Auniņš Endzelins zu entgegnen wußte: wenn man Usinšs auch die Funktionen eines Bienengottes zuerkannte, gab es im Bewußtsein des Volkes, für das Usinš der Bringer aller Wohltaten des Frühlings war, keine Schwierigkeit, in diese auch die Bienen einzubeziehen. Das war eine richtige, aber unnötige Erklärung, denn wie wir wissen, ist der behoste Bienengott ein später Pseudogott. Er fand in den Quellen keinerlei Begründung ebensowenig wie die spekulativen, auf phonetischem Gleichklang beruhenden Behauptungen von einem schnurrbärtigen Gott Ūsinšs.

5.4.4. Das zuletzt Gesagte läßt noch zwei Fragen offen. Einerseits ist es der verständliche Wunsch, Usiņš von den übrigen Himmelsgöttern noch genauer abzugrenzen. Andererseits kann man das Verlangen nach einer bestimmteren Begründung verstehen, warum gerade dieser Himmelsgott zu einem so ausgesprochenen Pferdepatron geworden ist.

5.4.4.1. Es haben wohl schon mehrere Forscher versucht, die erste Frage zu beantworten, und daher auch viel bestimmtere Antworten gegeben, als wir sie in dieser Arbeit finden. Zuerst ist es Bielenstein, der Ūiņš mit der Frühlingssonne identifiziert ${ }^{1}$. Seine einzige Begründung sind unsere Texte über Usiņš der hinter dem Stall , richtete sich hoch auf " und tanzt. Das Merkmal der Frühlingssonne sei es, daß sie aufgeht und höher steigt. Wie wir gesehen haben, bieten diese Texte dennoch keine ausreichende Grundlage für so weitgehende und sichere Behauptungen. Nicht weniger gewagte Behauptungen hat Schröder ausgesagt. Wie wir gehört haben, identifizierte er Usiņš völlig mit der Sonne. Er hat dieselben Texte wie Bielenstein verwendet und quellenmäßig nichts Neues gebracht. Zu beiden

zum ersten Mal auf die Weide zu treiben. Der Autor dieser Arbeit kann aus seinen Kindheitserinnerungen in der Gemeinde Lestene Anfang der zwanziger Jahre dieses Jahrhunderts bezeugen, daß der erste Tag des Viehaustriebs auf die Weide Festtagscharakter hatte. Der Brauch des Reitens zur Nachthütung war um jene Zeit bereits verschwunden.

1 Cf. Auniņš 1881, 25; 1905, 18. 
Ansichten ist zu bemerken, daß es außer den vorhin dargestellten Schwierigkeiten in den Quellenaussagen noch ein anderes, sogar viel größeres Hindernis gibt. Saule steht als Göttin im Mittelpunkt der Religion der alten Letten. Sie besitzt selbst Pferde und ist als Fahrerin und Reiterin über den Himmelsberg weit bekannt. Wenn man sie mit Ǔsiņ̌s identifizieren wollte, müßte man dafür ein besonderes Motiv haben. Keiner dieser Forscher hat eines genannt. Grundsätzlich wäre es denkbar, aber dann müßte man von einer Abtrennung einer besonderen Funktion der Saule sprechen, was einer Hypostase als selbständiger Gott gleichkommen würde. Das erscheint nicht glaubhaft. Die Schwierigkeiten würden noch dadurch wachsen, daß man in den Quellen nachweisen müßte, wo Saule ihre Funktionen allein ausübt und wo sie gewisse Funktionen an Usiņ̌s weitergegeben hat. Das ist jedoch nicht möglich. Die Identität von Saule und Üsinš ist nicht begründet. Es ist eine Hypothese, die seinerzeit unter dem Einfluß der Sonnenmythologie entstanden ist.

5.4.4.2. Bielenstein hat seine Ansicht über die Identität von Ūsiņš mit der Frühlingssonne unter dem direkten Einfluß von Bezzenberger geäußert. Auniņš beruft sich auf einen Brief, den jener an Bielenstein geschrieben hat, in dem es heißt: „Der Frühlingsgott Uhsingsch ist etymologisch zweifellos mit der Ostara verwandt. Grundform des letzteren Wortes ist ausra =lith. auszra. Seine Stellung in der vergleichenden Mythologie scheint mir damit darnach gegeben zu sein." ${ }^{1}$ Ohne nochmals auf die abgelehnten etymologischen Zusammenhänge zurückzukommen, ist nur die Tatsache zu erwähnen, daß Bezzenberger diesen Gedanken ausgesprochen hat. Er hat ihm keine weitere Begründung in den Quellen gegeben. Doch ist Bielenstein anscheinend dadurch ermuntert worden, Usingš mit der Frühlingssonne zu identifizieren.

Von Bezzenbergers Frühlingsgott und Bielensteins Frühlingssonne war es nur ein kleiner Schritt bis zur nächsten Behauptung von Endzelīns: „In manchen von Auniņ̌ aufgeschriebenen Volksliedern erscheint Ūsiņ̌ uns als Personifizierung des 23. April und damit des Frühlings. "'2 Als Begründung beruft er sich auf die Lieder 3, 5, 6 u. a. Es stimmt, daß die Kalendertage in den Volkstraditionen personifiziert werden. Dagegen gibt es keine Beweise, daß der jetzige Tag des 23. April des christlichen Kalenders ursprünglich mit dem Namen von Usiṇ,s bzw. dem Trühlingsgott verbunden gewesen ist. Daß das nicht der Fall ist, kann man so klar erkennen, daß diese Frage deshalb in dieser Arbeit auch nicht erörtert wurde ${ }^{3}$. Abgesehen

\footnotetext{
I Ib. 39. Auch Krause 1893, 216, spricht von einem, ,Gott des Frühlings“c.

2 Endzelins 1903, 26.

3 Das wurde seinerzeit bereits von Adamoviěs 1937, 95, abgelehnt. Cf. auch Volkslieder 24.
} 
von dem Namen des Ūsinšs in Verbindung mit dem 23. April, bleibt noch der Gedanke von Endzelīns der Personifizierung des Frühlings. Leider hat er ihn weder weiter begründet noch als richtig bestätigt.

5.4.4.3. Wie wir bereits wissen, hat nur Auniņš ein umfangreicheres, selbst gesammeltes Folklorematerial erforscht sowie auch eine sprachwissenschaftliche Analyse und historische Dokumentation dieses Materials vorgenommen. Seine Ansicht hat er sehr bestimmt geäußert: „Das Etymon des Uhßing-Namens nötigt uns zu dem Schlusse, daß der Uhßing ursprünglich ein Lichtgott gewesen sein muß. Wo Licht ist, da ist auch Wärme und Leben. So erklären sich uns auch viele der Prädicate die dem UhBing in den mitgeteilten Uhßing-Liedern beigelegt werden. "I Zunächst ist $\mathrm{zu}$ dieser Behauptung zu sagen, daß Auniņš sie, ebenso wie die vorhin erwähnten Bielenstein, Schröder u. a., auf einer irrigen etymologischen Deutung des Namens Ūsiņš, wie wir sie dem Zitat entnehmen können, begründet hat. Sein eigenes veröffentlichtes Material scheint ihm von sekundärer Bedeutung zu sein. Es dient nur zur Erläuterung der etymologisch begründeten Ansicht. Jedenfalls ist das der schwache Punkt in Auniņs' Argumentation. Ferner ist zu seiner Behauptung, Osiņš sei der Lichtgott, zu bemerken, daß im ganzen Material nicht eine einzige direkte Aussage zu finden ist, in der Ūsinš als Lichtgott bezeichnet wird. Ebenfalls fehlt jede direkte Aussage, die Usiņš mit dem Licht in Beziehung setzen würde. Das bedeutet aber nicht daß überhaupt jede Grundlage fehlen würde, Ūsinšs als Lichtgott zu bezeichnen, im Gegenteil - Auniņš hat das Wesen der Erscheinung richtig erkannt². Man muß seine Situation in Betracht ziehen. Er mußte im Jahre 1881, lange vor der Entwicklung einer modernen Terminologie in der Religionswissenschaft und vor der Anwendung phänomenologischer und typologischer Methoden, eine adäquate Bezeichnung finden. Heute ist es möglich, seiner Erkenntnis von Ūiṇš als Lichtgott methodisch eine ganz andere Grundlage zu geben. Usiņš gehört typologisch zu den Himmelsgöttern. Inhalt und Art der Quellen lassen in seinem Wesen nur phänomenologisch solche Züge erkennen, die es erlauben, ihn mit den Erscheinungen des Lichtes zu verbinden. Nur wenn man sich zu den Eigenheiten und Bedingungen der phänomenologischen und typologischen Methoden bekennt und sie beachtet, kann man von Ūsiņ als einem Lichtgott sprechen, ungeachtet dessen, daß in den Quellen diese direkte Bezeichnung fehlt. Unsere Quellenanalyse erlaubt es - man kann eher sagen, zwingt dazu -, ihn phänomenologisch eindeutig als Lichtgott anzusehen. Doch ebenso deutlich ist als charakteristisch für eine derartige methodisch begründete Erkenntnis

\footnotetext{
1 Auniñš 1881, 40. Ähnlich auch 1905, 14, $29 \mathrm{~s} ; 1913,27$.

2 Diese Auffassung von Auniṇš haben Volteris, Kiparts, Lautenbachs u. a. übernommen, ohne daß sie ihr eine neue und eingehendere Begründung gegeben hätten.
} 
festzustellen, daß es für die Bezeichnung von Ūsiņš selbst als Lichtgott keine historische Dokumentation gibt.

Nach dieser Erkenntnis wird auch verständlich, wieso die Forscher so verschiedene Ansichten äußern konnten. Die differenzierten, genaueren Anschauungen, daß Ūsinšs der Sonnengott, die Frühlingssonne oder der personifizierte Frühling sei, verlangen weitere spezifische Merkmale, die es in den Quellen nicht gibt. Dagegen ist die Bezeichnung "Lichtgott" oder „Lichtgottheit" umfassender. Sie bietet die Möglichkeit, entsprechend der Situation hier den einen, dort den anderen Zug im Wesen und in den Funktionen dieses Gottes zu betonen. Das ist der Grund, weshalb ich in dieser Arbeit bei dieser Bezeichnung geblieben bin'1.

5.4.4.4. Die andere Frage, die, wie bemerkt, noch einer Antwort harrt, Iautet: Weshalb ist dieser Lichtgott zu einem ausgesprochenen Pferdepatron geworden? Hier, scheint es, kommen zwei Umstände in Betracht. Es ist vor allem die in dieser Arbeit mehrfach erwähnte Tatsache, daß alle lettischen Himmelsgötter mit Pferden in Beziehung stehen. Das ist ein tief verwurzelter Wesenszug, der sogar die Tendenz aufweist, auch solche Götter mit einzubeziehen, die ursprünglich mit den Himmelsgöttern keinen Zusammenhang hatten, wie z. B. Laima. Die Beziehung der Himmelsgötter zu Pferden ist eine sehr alte indoeuropäische Volkstradition, und die Letten bilden hierbei keine Ausnahme. Eher hat umgekehrt deren späte Christianisierung es ermöglicht, daß diese Elemente sich in so ausgesprochener Weise auch in späterer Zeit erhalten haben.

Diese Umstände reichen an und für sich schon aus, um die Ansichten zu bezweifeln, daß Ūsiņš sich unter dem Einfluß des hl. Georg zum Pferdegott entwickelt habe. Auf jeden Fall war die hervorragende Rolle des Pferdes in dieser bäuerlichen Kultur schon lange vor Erscheinen des Christentums begründet. Das Pferd war in dieser Kultur schon längst in eine mythische Sphäre erhoben und gehörte zur sakralen Welt. Diese Erkenntnis darf allerdings nicht zur Grundlage für eine irrige Ansicht in entgegengesetzter Richtung werden, daß dem Kult des hl. Georg in dem so ausgesprochenen Pferdepatronat des Usinšs keine Bedeutung zugekommen wäre. Wahrscheinlich kommen wir der Wahrheit näher, wenn wir behaupten, daß die Rolle des hl. Georg sehr groß war. Das läßt sich auch so verstehen, daß seine Rolle im christlichen Synkretismus in bezug auf Ūsinšs eine bewahrende gewesen ist. Er, der in der Zeit der Christianisierung der Letten als Reiter bekannt war, verhalf dazu, daß sich die Vorstellungen von einem anderen Reiter, der einer ganz anderen religiösen Welt angehörte, erhielten. Wir

1 Wenn man von der oben behandelten linguistischen Analyse des Namens Üsinšs von Schmid ausgeht, stützt auch sie diese Auffassung (cf. Schmid 137). Dann würde dem nichts entgegenstehen, diesen Gott auch mit dem Namen Ūsin̨š zu bezeichnen. 
treffen hier auf ein bekanntes Modell im Wandlungsprozeß des lettischen religiösen Synkretismus, daher sei hieran erinnert.

In der Religion der Letten bezieht sich eine der ältesten Vorstellungen auf die Dieva dēli, 'Gottessöhne', ebenfalls hervorragende Reiter, die den altindischen Açvin entsprechen'. Das Christentum trat mit der Nachricht vom Gottessohn, wenn auch anders motiviert, auf, einer wohlbekannten empirischen Erscheinung, aber auch einer religiösen Vorstellung bei den Letten selbst. Doch war die religiöse Kraft der alten Gottessöhne so groB, daß man den christlichen Gottessohn, als sich der Synkretismus entwickelte, ohne weiteres in den Kreis der lettischen Gottessöhne aufnahm und ihm auch deren Funktionen zugestand. Und nicht nur Jesus allein, sondern eine ganze Reihe christlicher Apostel und Heiliger wurde zu Gottessöhnen². Andererseits aber gab der christliche Gottessohn einen Teil seiner Funktionen und Attribute auch an die alten lettischen Gottessöhne ab, nämlich das Kreuz, die Kerze u. a. Nach diesem Modell, scheint es, haben sich die synkretistischen Vorstellungen von U̦inšs dem alten Lichtgott, gebildet. Er reitet auf seinem steinernen Pferd im Frühling über den Himmelsberg herbei, bringt den Bäumen Laub und den Pferden Gras. Damit sichert er die Existenz des Bauers. Um im Bilde dieser Metaphern zu bleiben: jetzt verstehen wir, daß der lettische Bauer nach dem Opfermahl seine Pferde nicht in die Obhut des hl. Georg oder des christlichen Gottes stellte, wie man es im 19. Jh. erwarten konnte, sondern sie dem großen Piegulnieks, 'Nacht-

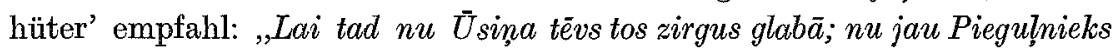
$m a \bar{j} \bar{a}$ ! 'So möge denn Vater Ūsinšs die Pferde beschützen; jetzt ist ja der Nachthüter zu Hause!'“ Zu diesem Gebet des lettischen Bauers ist zu bemerken, daß der semantische Sinn des letrten Wortes ,zu Hause" „,ist gekommen" bedeutet. Der alte Lichtgott war in nächtlicher Dunkelheit vom Himmelsberg zum lettischen Bauern hergeritten, und dieser fühlte sich in dessen Schutz sicher.

1 Cf. Biezais $1972,467 \mathrm{ss}$.

2 Hier genügt die Erwähnung des folgenden Textes:

Dievinam četri dēli, Es visiem värdus zinu: Mikušini, Andrušini, Pēterī̌si, Pāvulī̌š̀. Dievinam četri dēli, Ko tie tam labu dara? Zīda plavas neplaunamas, Zelta kalni nearami. 33734, 2
Gott dim. hat vier Söhne, ich kenne von allen die Namen: Michael dim. $p l$., Andreas dim. pl., Peter dim. pl., Paul dim. pl. Gott dim. hat vier Söhne, was tun sie ihm Gutes? Seidene Wiesen sind nicht zu mähen, goldene Berge sind nicht zu pflügen.

Weiter über das Problem bei Biezais 1972, 440 ss. 